\title{
Two-Stage Ordering Policy under Buyer's Minimum-Commitment Quantity Contract
}

\author{
Hsi-Mei Hsu, Zi-Yin Chen \\ Department of Industrial Engineering \& Management, National Chaio Tung University, \\ Hsinchu, Chinese Taipei \\ E-mail: hsimei@cc.nctu.edu.tw,yahuilin2@ydu.edu.tw \\ Received July 1, 2011; revised July 22, 2011; accepted August 16, 2011
}

\begin{abstract}
In this paper we consider a two-stage ordering problem with a buyer's minimum commitment quantity contract. Under the contract the buyer is required to give a minimum-commitment quantity. Then the manufacturer has the obligations to supply the minimum-commitment quantity and to provide a shortage compensation policy to the buyer. We formulate a dynamic optimization model to determine the manufacturer's two stage order quantities for maximizing the expected profit. The conditions for the existence of the optimal solution are defined. And we also develop a procedure to solve the problem. Numerical examples are given to illustrate the proposed solution procedure and sensitivity analyses are performed to find managerial insights.
\end{abstract}

Keywords: Two Stages Ordering, Commitment, Bayesian Information Updating

\section{Introduction}

In this paper we study a two-stage component ordering problem with a buyer minimum-commitment quantity contract. Under the contract, the buyer is required to commit a minimum order quantity $\theta_{1}$ and for returning the buyer's commitment the manufacturer has the obligations to supply the minimum-commit quantity $\theta_{1}$ and to give shortage compensation if the manufacturing supply level is under $(1+\gamma) \theta_{1}$ where $\gamma$ is a shortage compensation range coefficient $0 \leq \gamma \leq 1$. Because of the presence of the long lead time of key components, the manufacturer has two opportunities to place his order to supplier before the buyer's demand realized.

The buyer's real demand $X$ is uncertain following a normally distribution $N\left(\mu_{0}, \sigma_{0}^{2}\right)$ where $\mu_{0}$ is uncertain having a normal distribution $N\left(\mu, \sigma_{1}^{2}\right)$. When the manufacturer makes his first order quantity $\left(q_{1}\right)$ decision at stage 1 , the unit cost of key component at stage $1 c^{(1)}$ is known but the unit cost $C^{(2)}$ at the stage 2 is uncertain. The possible values of $C^{(2)}$ and their corresponding probabilities are known, denoted as

$C^{(2)}=\left\{c_{1}^{(2)}, c_{2}^{(2)}, \cdots, c_{n}^{(2)}\right\}$ and $P=\left\{p_{1}, p_{2}, \cdots, p_{n}\right\}$ respectively. After receiving the buyer's minimum-commit quantity $\theta_{1}$, the manufacturer uses $\theta_{1}$ as an estimator of $\mu$ and places his first order quantity $\left(q_{1}\right)$ to his supplier. At stage 2 the marketing department provides an observation $\theta_{2}$ of $X$. The posterior distribution of $X$ is defined by the observations of $\theta_{1}$ and $\theta_{2}$. Then manufacturer places his second order quantity $q_{2}$ if necessary. The time events of key component procurement process are shown in Figure 1.

We assume that the outputs are mainly limited by the available amounts of the key component, and the production cycle times are very short that can be neglected. After receiving the key components, the manufacturer produces the products immediately. Products are delivered to the buyer at the end of period (immediately after second stage). Due to the demand uncertainty, the manufacturer is difficult to determine two stage order quantities.

In this paper we develop a two-stage dynamic optimization model to decide the order quantity of a key component under a buyer's minimum-commitment quantity contract. The model is formulated to maximize a manufacturer's profit. The following costs are considered in the model. 1) Key component unit cost: the unit cost of key component at stage 1 is $c^{(1)}$ and the unit cost at stage 2 is $C^{(2)}$.2) Holding cost: two kinds of inventories are considered. One is buyer responsible inventory, which only exists in the case of buyer's real demand $(x)$ below the minimum guaranteed quantity $\theta_{1}$. In this case, customers only take away real demand $x$, and the remaining products $\left(\theta_{1}-x\right)$ are buyer responsible inventory 


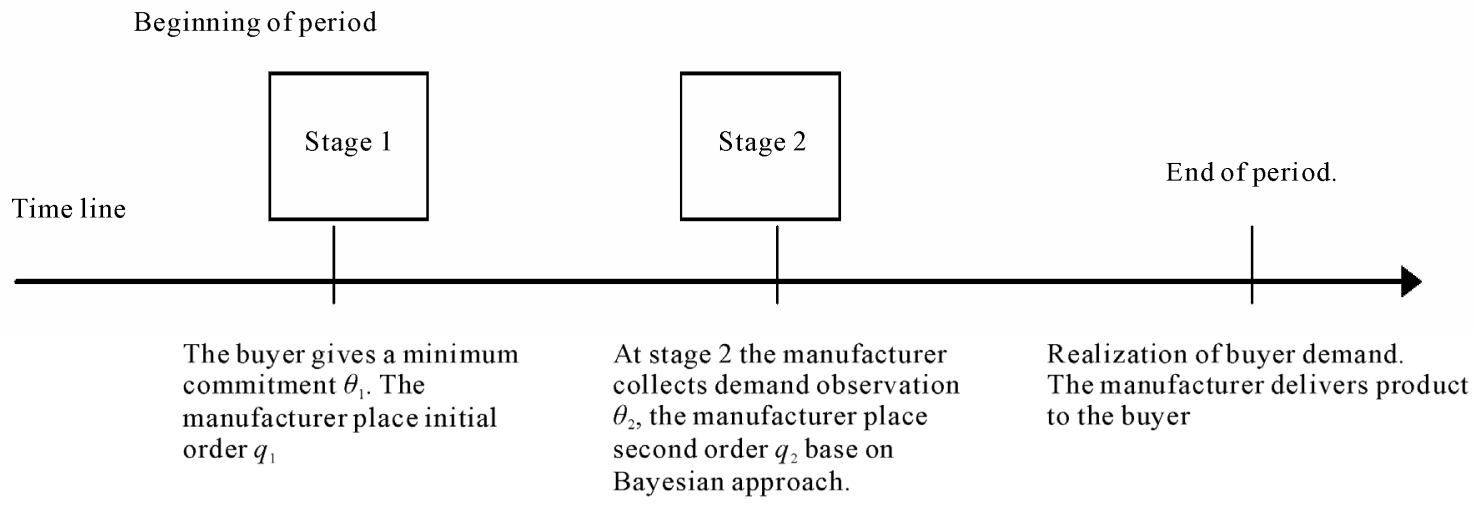

Figure 1. Time events of key component procurement process.

and will be paid in the near future. The unit holding costs of buyer responsible inventory are the interest and insurance. The other is manufacturer responsible inventory. The unit holding cost of manufacturer responsible inventory is the interest, insurance and obsolete costs. The holding costs of buyer responsible inventory and manufacturer responsible inventory are $c_{h 1}$ and $c_{h 2}$ respectively and $c_{h 2}>c_{h 1}$. 3) Shortage cost: two kinds of shortage cost are considered according to whether or not to pay shortage compensation. If the manufacturing output level is below $(1+\gamma) \theta_{1}$, then there are two shortage types may occurred. The one includes general shortage cost and the compensation cost. The other is only the general shortage cost.

The buyer's minimum commitment and demand forecast updating in this paper belong to the category of minimum purchase commitment contract [1] and inventory management with demand forecast updates respectively [2]. Durango-Cohen and Yano [3] pointed out that increasing the level of commitment and information sharing will lead to the cost down of entire supply chain. Nowadays minimum commitment quantity contracts are commonly used in electronic industry. Anupindi and Bassok [1] classified the contract of quantity commitments and flexibility as three types. The first type is the total minimum quantity commitment contract. The supply contract with total minimum quantity commitment is that a buyer gives his supplier a minimum ordering quantity commitment, and the supplier offers the buyer a discount price in return for the buyer's commitment [4]. The second type is the total minimum dollar volume commitment contract. This contract is similar to the total minimum quantity commitment contract, but a buyer commits to a minimum business on the basis of dollar volume [5], and the supplier offers discounts based on the commitment of dollar volume. The third type is the periodical commitment with flexibility contract. Under such a contract, a buyer receives discounts for committing to purchase in advance, and the buyer is allowed to update his order amount in the rolling horizon basis. The rolling horizon flexibility (RHF) contract [6-8] is one kind of the third type. The RHF contract means the buyer has a "limited" flexibility to update his advance order after he commits to purchase certain quantity.

Gallego and Ozer [9] and Sethi et al. [2] classified the inventory information with demand updating problems as three types. The first type is the Bayesian analysis. This approach learns about further demand from the past history [10]. Dvoretzky et al. [11] first analyzed Bayesian models in the inventory problem. In this type, specific classes of demand distribution were discussed, such as exponential family of distribution [12], gamma family $[13,14]$, negative binomial distribution [15], uniformPareto distribution [16] and normal distribution [17,18]. The second type is time-series models used in updating demand forecast, where they assume a correlation exists in the demand realization and construct the demand as a time-series model $[10,19]$. The third type is concerned with forecast revisions, such as Markovian forecast revisions model [20-22], single-period, two-stage ordering problem with demand forecast updating [23-26] and multiple period ordering problem with demand forecast updating $[27,28]$. A more comprehensive discussion can be found in [2].

Our study differs from the previous papers because we consider a shortage compensation policy for reducing demand uncertainty. Under the buyer's minimum-commitment quantity contract and shortage compensation policy, two kinds of inventory and shortage costs are respectively formulated in the two-stage dynamic optimization model to decide the optimal order quantities.

In this paper, we define the conditions for the existence of the optimal solution and also develop a procedure to determine the two-stage optimal order quantities. The rest of this paper is organized as follows: Section 2 states the assumptions and notations for the proposed model; the two-stage dynamic optimization model and the solution procedure are proposed. Section 2 illustrates 
some numerical examples, and sensitivity analyses of the major parameters of the model are performed. Finally, Section 4 concludes this article.

\section{Problem Formulation}

\subsection{Notations}

$\theta_{1}$ : buyer's minimum-commitment quantity. $\theta_{1}>0$

$\gamma$ : shortage compensation range coefficient $0 \leq \gamma \leq 1$.

$\left[\theta_{1},(1+\gamma) \theta_{1}\right]$ is the shortage compensation range.

$c^{(1)}$ : unit ordering cost of key component at stage 1 .

$C^{(2)}$ : unit ordering cost of key component at stage 2 is a random variable, $C^{(2)}=\left\{c_{1}^{(2)}, c_{2}^{(2)}, \cdots, c_{n}^{(2)}\right\}$ and the corresponding probability $P=\left\{p_{1}, p_{2}, \cdots, p_{n}\right\}$.

$p$ : product unit selling price. $p>c^{(1)}$.

$\theta_{2}:$ the demand observation at stage 2 .

$c_{h 1}$ : unit holding cost of buyer responsible inventory.

$c_{h 2}$ : unit holding cost of manufacturer responsible inventory.

$c_{s 1}$ : unit shortage compensation cost; $c_{s 1}>0$

$c_{s 2}$ : unit general shortage cost; $c_{s 1} \geq c_{s 2}>0$

$X$ : buyer's real demand, realization is denoted by ${ }^{X}$.

$X^{(1)}: X^{(1)}$ is a random variable to forecast the buyer's demand at stage $1, X^{(1)}=X \mid \theta_{1}$.

$X^{(2)}: X^{(2)}$ is a random variable to forecast the buyer's demand at stage $2, \quad X^{(2)}=X \mid \theta_{1}, \theta_{2}$.

$f_{1}($.$) : probability density function (pdf) of X^{(1)}$, $X^{(1)} \sim N\left(\theta_{1}, \sigma_{0}^{2}+\sigma_{1}^{2}\right)$

$f_{2}\left(\right.$.) : probability density function (pdf) of $X^{(2)}$,

$$
\begin{gathered}
X^{(2)} \sim N\left(\left[\sigma_{1}^{2} \theta_{2} /\left(\sigma_{0}^{2}+\sigma_{1}^{2}\right)+\sigma_{0}^{2} \theta_{1} /\left(\sigma_{0}^{2}+\sigma_{1}^{2}\right)\right],\right. \\
\left.\sigma_{0}^{2}+\sigma_{1}^{2} \sigma_{0}^{2} /\left(\sigma_{0}^{2}+\sigma_{1}^{2}\right)\right)
\end{gathered}
$$

$F_{1}($.$) : cumulative density function (cdf) of. X^{(1)}$

$F_{2}($.$) : cumulative density function (cdf) of X^{(2)}$.

$\phi($.$) : standard normal probability density function.$

$\Phi($.$) : the cumulative distribution function for standard$ normal distribution.

$\Phi^{-1}($.$) : inverse function of \Phi($.$) .$

$\Psi($.$) : the standard linear loss function:$

$$
\Psi(a)=\int_{a}^{\infty}(x-a) \mathrm{d} \Phi(x) .
$$

Decision variables:

$q_{1}$ : order quantity at stage $1 . q_{1} \geq 0$

$q_{2}$ : order quantity at stage $2 . q_{2} \geq 0$

Intermediate variables:

$\Omega_{1}$ : the decision space defined by $\theta_{1} \leq q_{1}+q_{2} \leq(1+\gamma) \theta_{1}$.

$\Omega_{2}$ : the decision space defined by $(1+\gamma) \theta_{1}<q_{1}+q_{2}$.

$q_{11}^{*}$ : optimal order quantity in $\Omega_{1}$ at stage 1

$q_{21}^{*}$ : optimal order quantity in $\Omega_{1}$ at stage 2

$q_{12}^{*}$ : optimal order quantity in $\Omega_{2}$ at stage 1 $q_{22}^{*}$ : optimal order quantity in $\Omega_{2}$ at stage 2

$q_{1}^{*}$ : optimal order quantity at stage 1

$q_{2}^{*}$ : optimal order quantity at stage 2

\subsection{Problem Assumptions and Formulation}

The mathematical model is formulated to determine the two stage ordering quantities of the key component for maximizing profit. The buyer's real demand $X$ is uncertain to be assumed following a normal distribution with an uncertain mean $\mu_{0}$ and a given variance $\sigma_{0}^{2}$, where $\mu_{0}$ follows $N\left(\mu, \sigma_{1}^{2}\right)$ with an unknown $\mu$ and a given variance $\sigma_{1}^{2}$. At stage 1 , after receiving the buyer's minimum commitment quantity $\theta_{1}$, the manufacturer uses $\theta_{1}$ as an estimator of $\mu$. The posterior distribution of $X$ after receiving $\theta_{1}$ at stage 1 is denoted as $X^{(1)}=X \mid \theta_{1}$ where

$$
X^{(1)}=X \mid \theta_{1} \sim N\left(\theta_{1}, \sigma_{0}^{2}+\sigma_{1}^{2}\right)
$$

At stage 2, the marketing department collects information $\theta_{2}$ about buyer's real demand. We call it as an observation of $X$. The posterior distribution of $X$ at stage 2 is denoted as $X^{(2)}=X \mid \theta_{1}, \theta_{2}$ where

$$
\begin{gathered}
X^{(2)}=X \mid \theta_{1}, \theta_{2} \sim N\left(k_{2}, \sigma_{2}^{2}\right) \\
k_{2}=\left[\sigma_{1}^{2} \theta_{2} /\left(\sigma_{0}^{2}+\sigma_{1}^{2}\right)+\sigma_{0}^{2} \theta_{1} /\left(\sigma_{0}^{2}+\sigma_{1}^{2}\right)\right] \\
\sigma_{2}^{2}=\sigma_{0}^{2}+\sigma_{1}^{2} \sigma_{0}^{2} /\left(\sigma_{0}^{2}+\sigma_{1}^{2}\right)
\end{gathered}
$$

Because the manufacturer has the obligation to provide the minimum-commitment quantity $\theta_{1}$ to the buyer, the total order quantities $q_{1}+q_{2}$ must be larger than $\theta_{1}$. Now we will formulate the expected profit function and use a backward dynamic programming to determine the optimal $q_{1}^{*}$ and $q_{2}^{*}$.

We formulate the problem as a dynamic programming (DP) problem. For the DP formulation, the ordering times are given as the stages, stage 1 and stage 2 . Decision variable for stage $n(n=1,2)$ is the ordering quantity $q_{n}$. The profit at the current stage depends upon the current decision $q_{n}$ and the ordering quantity in the preceding stage $q_{n-1}$. We set states for each stage $n$ as $q_{n-1}$.

With the backward solving procedure, first we should determine the optimal order quantity $q_{2}$ at stage 2 in a given state $s_{2}=q_{1}$. We denote the expected profit function at stage 2 as $E_{(2)}\left[\Pi\left(q_{2} \mid q_{1}\right)\right]$. The state at stage 1 is known as $s_{1}=0$. The expected profit function at stage 1 is denoted as $E_{(1)}\left[\Pi\left(q_{1}\right)\right]$, where

$$
E_{(1)}\left[\Pi\left(q_{1}\right)\right]=E\left[-c^{(1)} q_{1}+E_{(2)}\left[\Pi\left(q_{2}^{*} \mid q_{1}\right)\right]\right]
$$


The optimal expected profit of the manufacturer is determined as follows:

$$
\begin{aligned}
E_{(1)}\left[\Pi\left(q_{1}^{*}\right)\right] & =\max _{q_{1} \geq 0} E_{(1)}\left[\Pi\left(q_{1}\right)\right] \\
& =\max _{q_{1} \geq 0} E\left[-c^{(1)} q_{1}+E_{(2)}\left[\Pi\left(q_{2}^{*} \mid q_{1}\right)\right]\right]
\end{aligned}
$$

where

$$
E_{(2)}\left[\Pi\left(q_{2}^{*} \mid q_{1}\right)\right]=\max _{q_{2} \geq 0} E_{(2)}\left[\Pi\left(q_{2} \mid q_{1}\right)\right]
$$

The items considered in $E_{(2)}\left[\Pi\left(q_{2} \mid q_{1}\right)\right]$ are expected product sales, ordering costs, expected holding costs and expected shortage costs.

$$
\begin{aligned}
& E_{(2)}\left[\Pi\left(q_{2} \mid q_{1}\right)\right]=\text { Expected revenues } \\
& \text { - Expected costs } \\
& =\text { Expected product sales } \\
& -(\text { Ordering costs }+ \text { Expected holding costs } \\
& + \text { Expected shortage costs })
\end{aligned}
$$

The relevant items are formulated respectively as fol- lows:

1) Expected products sales

$$
=\int_{X}\left\{p \times \min \left\{x, q_{1}+q_{2}\right\} f_{2}(x) \mathrm{d} x\right.
$$

2) Ordering costs when $C^{(2)}=c^{(2)}$

$$
c^{(2)} q_{2}
$$

3) Expected holding costs:

The unit holding cost of buyer responsible inventory is $c_{h 1}$ and holding cost of manufacturer responsible inventory is $c_{h 2}$. The holding costs can be expressed as follows:

$$
\left\{\begin{array}{cc}
c_{h 1} \times\left(\theta_{1}-x\right)+c_{h 2} \times\left(q_{1}+q_{2}-\theta_{1}\right) & \text { for } x<\theta_{1} \\
c_{h 2} \times\left(q_{1}+q_{2}-x\right) & \text { for } \theta_{1}<x<q_{1}+q_{2} \\
0 & \text { otherwise }
\end{array}\right.
$$

The expected holding costs can be formulated as follows:

$$
\int_{X}\left\{c_{h 1} \times \max \left\{\theta_{1}-x, 0\right\}+c_{h 2} \times\left\{q_{1}+q_{2}-\max \left\{\theta_{1}-x, 0\right\}\right\}\right\} \times f_{2}(x) \mathrm{d} x
$$

4) Expected shortage costs:

The decision space of total order quantity $q_{1}+q_{2}$ can be divided into two subspaces $\Omega_{1}$ and $\Omega_{2}, \Omega_{1}$ : total order quantity $\left(q_{1}+q_{2}\right)$ is less than $(1+\gamma) \theta_{1}$, as shown in Figure 2(a); $\Omega_{2}$ : total order quantity $\left(q_{1}+q_{2}\right)$ is larger than $(1+\gamma) \theta_{1}$, as shown in Figure 2(b). In $\Omega_{1}$, two kinds of shortage types may occur: 1) the shortage occurred between $q_{1}+q_{2}$ and $(1+\gamma) \theta_{1}$ belongs to short- age compensation range, its unit shortage costs are the shortage compensation cost and general shortage cost; 2) the others does not belong to shortage compensation range, its unit shortage cost is general shortage cost. The unit shortage costs of shortage compensation and general shortage are $c_{s 1}$ and $c_{s 2}$ respectively, $c_{s 1}>c_{s 2}$. In $\Omega_{2}$, the shortage compensation cost does not occur. Two cases of shortage can be expressed as follows:

$$
\text { expected shortage costs in } \Omega_{1}=\left\{\begin{array}{cc}
c_{s 1} \times\left[x-\left(q_{1}+q_{2}\right)\right] & \text { for } q_{1}+q_{2}<x<(1+\gamma) \theta_{1}, \\
c_{s 1} \times\left[(1+\gamma) \theta_{1}-\left(q_{1}+q_{2}\right)\right]+c_{s 2} \times\left\{x-(1+\gamma) \theta_{1}\right\} & \text { for }(1+\gamma) \theta_{1}<x \\
0 & \text { otherwise }
\end{array}\right.
$$

expected shortage costs in $\Omega_{2}=\left\{\begin{array}{cc}c_{s 2} \times\left[x-\left(q_{1}+q_{2}\right)\right] & \text { for }(1+\gamma) \theta_{1}<q_{1}+q_{2}<x, \\ 0 & \text { otherwise. }\end{array}\right.$

Expected shortage cost can be combined as follows:

$$
\int c_{s 1}\left\{\max \left\{\min \left[(1+\gamma) \theta_{1}, x\right]-\left(q_{1}+q_{2}\right), 0\right\}+c_{s 2} \max \left\{x-\max \left\{\left(q_{1}+q_{2}\right),(1+\gamma) \theta_{1}\right\}, 0\right\}\right\} \times f_{2}(x) \mathrm{d} x
$$

\subsection{Optimal Solution}

To solve the DP problem we first provide the optimal decisions for stage 2 under a given state $s_{2}=q_{1}$. As mentioned above the expected shortage costs in
$E_{(2)}\left[\Pi\left(q_{2} \mid q_{1}\right)\right]$ depend on the domain of the total order quantity $q_{1}+q_{2}$, i.e., $\Omega_{1}=\left\{\left(q_{1}, q_{2}\right) \mid \theta_{1} \leq q_{1}+q_{2} \leq(1+\gamma) \theta_{1}\right\}$ and $\Omega_{1}=\left\{\left(q_{1}, q_{2}\right) \mid q_{1}+q_{2}>(1+\gamma) \theta_{1}\right\}$, each domain corresponds to a shortage cost equation respectively (Figures 2(a) and (b)). Therefore $E_{(2)}\left[\Pi\left(q_{2} \mid q_{1}\right)\right]$ is formulated 


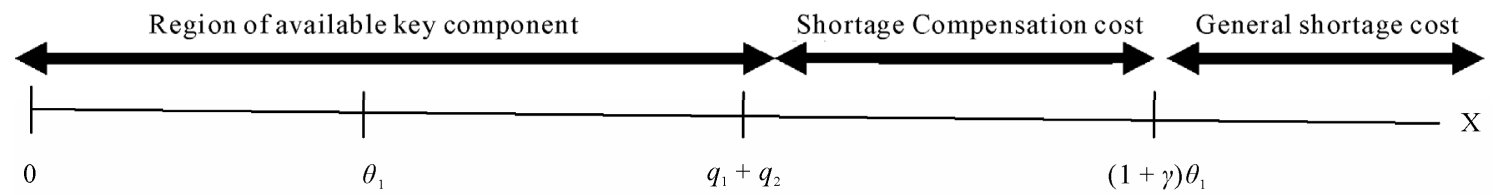

(a)

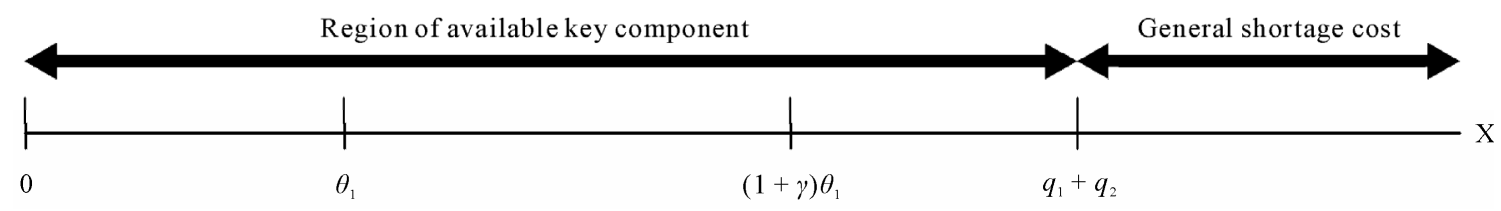

(b)

Figure 2. (a) in $\Omega_{1}$ : total order quantity $q_{1}+q_{2}$ is less than $(1+\gamma) \theta_{1}$; (b) in $\Omega_{2}$ : total order quantity $q_{1}+q_{2}$ is larger than $(1+\gamma) \theta_{1}$.

for each domain as follows:

$$
\begin{aligned}
E_{(2)}\left[\Pi\left(q_{2} \mid q_{1}\right)\right]_{\left(q_{1}, q_{2}\right) \in \Omega_{1}}= & \int_{-\infty}^{\theta_{1}} p \theta_{1} f_{2}(x) \mathrm{d} x+\int_{\theta_{1}}^{q_{1}+q_{2}} p x f_{2}(x) \mathrm{d} x+\int_{q_{1}+q_{2}}^{\infty} p\left(q_{1}+q_{2}\right) f_{2}(x) \mathrm{d} x-c_{h 1} \int_{-\infty}^{\theta_{1}}\left(\theta_{1}-x\right) f_{2}(x) \mathrm{d} x \\
& -c_{h 2} \int_{-\infty}^{\theta_{1}}\left(q_{1}+q_{2}-\theta_{1}\right) f_{2}(x) \mathrm{d} x-c_{h 2} \int_{\theta_{1}}^{q_{1}+q_{2}}\left(q_{1}+q_{2}-x\right) f_{2}(x) \mathrm{d} x \\
& -c_{s 1} \int_{q_{1}+q_{2}}^{(1+\gamma) \theta_{1}}\left(x-q_{1}-q_{2}\right) f_{2}(x) \mathrm{d} x-c_{s 2} \int_{(1+\gamma) \theta_{1}}^{\infty}\left(x-(1+\gamma) \theta_{1}\right) f_{2}(x) \mathrm{d} x-c^{(2)} q_{2}
\end{aligned}
$$

and

$$
\begin{aligned}
E_{(2)}\left[\Pi\left(q_{2} \mid q_{1}\right)\right]_{\left(q_{1}, q_{2}\right) \in \Omega_{2}}= & \int_{-\infty}^{\theta_{1}} p \theta_{1} f_{2}(x) \mathrm{d} x+\int_{\theta_{1}}^{q_{1}+q_{2}} p x f_{2}(x) \mathrm{d} x+\int_{q_{1}+q_{2}}^{\infty} p\left(q_{1}+q_{2}\right) f_{2}(x) \mathrm{d} x-c_{h 1} \int_{-\infty}^{\theta_{1}}\left(\theta_{1}-x\right) f_{2}(x) \mathrm{d} x \\
& -c_{h 2} \int_{-\infty}^{\theta_{1}}\left(q_{1}+q_{2}-\theta_{1}\right) f_{2}(x) \mathrm{d} x-c_{h 2} \int_{\theta_{1}}^{q_{1}+q_{2}}\left(q_{1}+q_{2}-x\right) f_{2}(x) \mathrm{d} x \\
& -c_{s 2} \int_{q_{1}+q_{2}}^{\infty}\left(x-q_{1}-q_{2}\right) f_{2}(x) \mathrm{d} x-c^{(2)} q_{2}
\end{aligned}
$$

We will show $E_{(2)}\left[\Pi\left(q_{2} \mid q_{1}\right)\right]_{\left(q_{1}, q_{2}\right) \in \Omega_{1}}$ and $E_{(2)}\left[\Pi\left(q_{2} \mid q_{1}\right)\right]_{\left(q_{1}, q_{2}\right) \in \Omega_{2}}$ are both concave functions. Then we can determine the optimal $q_{2}$ in the interval $[0, \infty)$ for the two cases respectively.

Proposition 1. If $p+c_{s 1}+c_{h 2}>0$ and $p+c_{s 2}+c_{h 2}>0$ hold for $q_{2} \in[0, \infty)$, then $E_{(2)}\left[\Pi\left(q_{2} \mid q_{1}\right)\right]_{\left(q_{1}, q_{2}\right) \in \Omega_{1}}$ and $E_{(2)}\left[\Pi\left(q_{2} \mid q_{1}\right)\right]_{\left(q_{1}, q_{2}\right) \in \Omega_{2}}$ are concave functions, i.e., $\frac{d^{2} E_{(2)}\left[\Pi\left(q_{2} \mid q_{1}\right)\right]_{\left(q_{1}, q_{2}\right) \in \Omega_{1}}}{d q_{2}^{2}} \leq 0$ and $\frac{d^{2} E_{(2)}\left[\Pi\left(q_{2} \mid q_{1}\right)\right]_{\left(q_{1}, q_{2}\right) \in \Omega_{2}}}{d q_{2}^{2}} \leq 0$ for $q_{2} \in[0, \infty)$.

Proof. See appendix A.
Proposition 2. Maximizing $E_{(2)}\left[\Pi\left(q_{2} \mid q_{1}\right)\right]_{\left(q_{1}, q_{2}\right) \in \Omega_{1}}$ and $E_{(2)}\left[\Pi\left(q_{2} \mid q_{1}\right)\right]_{\left(q_{1}, q_{2}\right) \in \Omega_{2}}$ with respect to $q_{2}$, we can get the optimal order quantity $q_{2}$ denoted as $q_{21}^{*}$ and $q_{22}^{*}$ for the two domains respectively as follows:

where

$$
q_{21}^{*}=\left\{\begin{array}{l}
\max \left\{0, k_{2}+\sqrt{\sigma_{0}^{2}+\sigma_{2}^{2}} \Phi^{-1}\left(t_{1}\right)-q_{1}\right\} \\
\quad \text { if } \theta_{1} \leq k_{2}+\sqrt{\sigma_{0}^{2}+\sigma_{2}^{2}} \Phi^{-1}\left(t_{1}\right) \leq(1+\gamma) \theta_{1} \\
\max \left\{0,(1+\gamma) \theta_{1}-q_{1}\right\} \\
\quad \text { if } k_{2}+\sqrt{\sigma_{0}^{2}+\sigma_{2}^{2}} \Phi^{-1}\left(t_{1}\right)>(1+\gamma) \theta_{1} \\
\max \left\{0, \theta_{1}-q_{1}\right\} \\
\quad \text { if } k_{2}+\sqrt{\sigma_{0}^{2}+\sigma_{2}^{2}} \Phi^{-1}\left(t_{1}\right)<\theta_{1}
\end{array},\right.
$$

$$
t_{1}=\left(p+c_{s 1} F_{2}\left((1+\gamma) \theta_{1}\right)-c^{(2)}\right) /\left(p+c_{h 2}+c_{s 1}\right)
$$




$$
q_{22}^{*}=\left\{\begin{array}{c}
\max \left\{0,(1+\gamma) \theta_{1}-q_{1}\right\} \\
\text { if } \theta_{1} \leq k_{2}+\sqrt{\sigma_{0}^{2}+\sigma_{2}^{2}} \Phi^{-1}\left(t_{2}\right) \leq(1+\gamma) \theta_{1} \\
\max \left\{0, k_{2}+\sqrt{\sigma_{0}^{2}+\sigma_{2}^{2}} \Phi^{-1}\left(t_{2}\right)-q_{1}\right\} \\
\text { if } k_{2}+\sqrt{\sigma_{0}^{2}+\sigma_{2}^{2}} \Phi^{-1}\left(t_{2}\right)>(1+\gamma) \theta_{1}
\end{array},\right.
$$

where

$$
t_{2}=\left(p+c_{s 2}-c^{(2)}\right) /\left(p+c_{h 2}+c_{s 2}\right)
$$

Proof: See appendix B.

Then we provide the optimal solution for stage 1 . At stage 1 , the profit functions

$$
\Pi\left(q_{1}\right)_{\left(q_{1}, q_{2}\right) \in \Omega_{1}}=-c^{(1)} q_{1}+E_{(2)}\left[\Pi\left(q_{21}^{*} \mid q_{1}\right)\right]_{\left(q_{1}, q_{2}\right) \in \Omega_{1}}
$$

and

$$
\begin{aligned}
\Pi\left(q_{1}\right)_{\left(q_{1}, q_{2}\right) \in \Omega_{2}}=-c^{(1)} q_{1}+E_{(2)}\left[\Pi\left(q_{22}^{*} \mid q_{1}\right)\right]_{\left(q_{1}, q_{2}\right) \in \Omega_{2}} & \begin{array}{l}
\Pi\left(q_{1}\right)_{\left(q_{1}, q_{2}\right) \in \Omega_{2}} \text { lows: } \\
\text { respectively, that are for }
\end{array} \\
E_{(1)}\left[\Pi\left(q_{1}\right)\right]_{\left(q_{1}, q_{2}\right) \in \Omega_{1}}= & \sum_{i=1}^{n} p_{i}\left(\int_{-\infty}^{q_{1}-\sqrt{d_{1}+d_{3}} \Phi^{-1}\left(t_{1}\right)} E_{(2)}\left[\Pi\left(q_{2}=0, q_{1}\right)_{\left(q_{1}, q_{2}\right) \in \Omega_{1}}\right] f_{K_{2}}\left(k_{2}\right) \mathrm{d} k_{2}\right. \\
& \left.+\int_{q_{1}-\sqrt{d_{1}+d_{3}} \Phi^{-1}\left(t_{1}\right)}^{\infty} E_{(2)}\left[\Pi\left(q_{2}>0, q_{1}\right)_{\left(q_{1}, q_{2}\right) \in \Omega_{1}}\right] f_{K_{2}}\left(k_{2}\right) \mathrm{d} k_{2}-c^{(1)} q_{1}\right) \\
= & \sum_{i=1}^{n} p_{i}\left(\int_{-\infty}^{q_{1}-\sqrt{d_{1}+d_{3}} \Phi^{-1}\left(t_{2}\right)} E_{(2)}\left[\Pi\left(q_{2}=0, q_{1}\right)_{\left(q_{1}, q_{2}\right) \in \Omega_{2}}\right] f_{K_{2}}\left(k_{2}\right) \mathrm{d} k_{2}\right. \\
& \left.+\int_{q_{1}-\sqrt{d_{1}+d_{3}} \Phi^{-1}\left(t_{2}\right)}^{\infty} E_{(2)}\left[\Pi\left(q_{2}>0, q_{1}\right)_{\left(q_{1}, q_{2}\right) \in \Omega_{2}}\right] f_{K_{2}}\left(k_{2}\right) \mathrm{d} k_{2}-c^{(1)} q_{1}\right)
\end{aligned}
$$

where

$$
\begin{aligned}
E_{(2)}\left[\Pi\left(q_{2}>0, q_{1}\right)_{\left(q_{1}, q_{2}\right) \in \Omega_{1}}\right]= & \left(p-c_{h 1}+c_{h 2}\right) \sqrt{\sigma_{0}^{2}+\sigma_{2}^{2}} \Psi\left(\left(\theta_{1}-k_{2}\right) / \sqrt{\sigma_{0}^{2}+\sigma_{2}^{2}}\right) \\
& -\left(p+c_{h 2}+c_{s 1}\right) \sqrt{\sigma_{0}^{2}+\sigma_{2}^{2}} \Psi\left(\Phi^{-1}\left(t_{1}\right)\right) \\
& +\left(c_{s 1}-c_{s 2}\right) \sqrt{\sigma_{0}^{2}+\sigma_{2}^{2}} \Psi\left(\left((1+\gamma) \theta_{1}-k_{2}\right) / \sqrt{\sigma_{0}^{2}+\sigma_{2}^{2}}\right) \\
& +\left\{p-c_{h 1}+c_{h 2}+c_{s 1}\left[1-\Phi\left(\left((1+\gamma) \theta_{1}-k_{2}\right) / \sqrt{\sigma_{0}^{2}+\sigma_{2}^{2}}\right)\right]\right\} \theta_{1} \\
& +\left\{c_{h 1}-c_{h 2}-c^{(2)}-c_{s 1}\left[1-\Phi\left(\left((1+\gamma) \theta_{1}-k_{2}\right) / \sqrt{\sigma_{0}^{2}+\sigma_{2}^{2}}\right)\right]\right\} k_{2} \\
& -\left\{c^{(2)}+c_{h 2}+c_{s 1}\left[1-\Phi\left(\left((1+\gamma) \theta_{1}-k_{2}\right) / \sqrt{\sigma_{0}^{2}+\sigma_{2}^{2}}\right)\right]\right\} \sqrt{\sigma_{0}^{2}+\sigma_{2}^{2}} \Psi\left(\Phi^{-1}\left(t_{1}\right)\right) \\
& +c_{s 1} \gamma \theta_{1}\left[1-\Phi\left(\left((1+\gamma) \theta_{1}-k_{2}\right) / \sqrt{\sigma_{0}^{2}+\sigma_{2}^{2}}\right)\right]+c^{(2)} q_{1}, \\
t_{1}=\left[p+c_{s_{1}} F_{2}\left((1+\gamma) \theta_{1}\right)-c^{(2)}\right. & ] /\left(p+c_{h 2}+c_{s 1}\right)
\end{aligned}
$$




$$
\begin{aligned}
& E_{(2)}\left[\Pi\left(q_{2}=0, q_{1}\right)_{\left(q_{1}, q_{2}\right) \in \Omega_{1}}\right]=-\left(p+c_{h 2}+c_{s 1}\right) \sqrt{\sigma_{0}^{2}+\sigma_{2}^{2}} \Psi\left(\left(q_{1}-k_{2}\right) / \sqrt{\sigma_{0}^{2}+\sigma_{2}^{2}}\right) \\
& +\left(p-c_{h 1}+c_{h 2}\right) \sqrt{\sigma_{0}^{2}+\sigma_{2}^{2}} \Psi\left(\left(\theta_{1}-k_{2}\right) / \sqrt{\sigma_{0}^{2}+\sigma_{2}^{2}}\right) \\
& +\left(c_{s 1}-c_{s 2}\right) \sqrt{\sigma_{0}^{2}+\sigma_{2}^{2}} \Psi\left(\left((1+\gamma) \theta_{1}-k_{2}\right) / \sqrt{\sigma_{0}^{2}+\sigma_{2}^{2}}\right) \\
& +\left\{p-c_{h 1}+c_{h 2}+c_{s 1}\left[1-\Phi\left(\left((1+\gamma) \theta_{1}-k_{2}\right) / \sqrt{\sigma_{0}^{2}+\sigma_{2}^{2}}\right)\right]\right\} \theta_{1} \\
& -\left\{c_{h 2}+c_{s 1}\left[1-\Phi\left(\left((1+\gamma) \theta_{1}-k_{2}\right) / \sqrt{\sigma_{0}^{2}+\sigma_{2}^{2}}\right)\right]\right\} q_{1} \\
& +c_{s 1}\left[1-\Phi\left(\left((1+\gamma) \theta_{1}-k_{2}\right) / \sqrt{\sigma_{0}^{2}+\sigma_{2}^{2}}\right)\right] \gamma \theta_{1}+c_{h 1} k_{2}, \\
& E_{(2)}\left[\Pi\left(q_{2}>0, q_{1}\right)_{\left(q_{1}, q_{2}\right) \in \Omega_{2}}\right]=\left(p-c_{h 1}+c_{h 2}\right) \sqrt{\sigma_{0}^{2}+\sigma_{2}^{2}} \Psi\left(\left(\theta_{1}-k_{2}\right) / \sqrt{\sigma_{0}^{2}+\sigma_{2}^{2}}\right) \\
& -\left(p+c_{h 2}+c_{s 2}\right) \sqrt{\sigma_{0}^{2}+\sigma_{2}^{2}} \Psi\left(\Phi^{-1}\left(t_{2}\right)\right)+\left(p-c_{h 1}+c_{h 2}\right) \theta_{1} \\
& +\left(c_{h 1}-c_{h 2}-c^{(2)}\right) k_{2}-\left(c^{(2)}+c_{h 2}\right) \sqrt{\sigma_{0}^{2}+\sigma_{2}^{2}} \Psi\left(\Phi^{-1}\left(t_{2}\right)\right)+c^{(2)} q_{1}, \\
& t_{2}=\left(p+c_{s 2}-c^{(2)}\right) /\left(p+c_{h 2}+c_{s 2}\right) \\
& E_{(2)}\left[\Pi\left(q_{2}=0, q_{1}\right)_{\left(q_{1}, q_{2}\right) \in \Omega_{2}}\right]=-\left(p+c_{h 2}+c_{s 2}\right) \sqrt{\sigma_{0}^{2}+\sigma_{2}^{2}} \Psi\left(\left(q_{1}-k_{2}\right) / \sqrt{\sigma_{0}^{2}+\sigma_{2}^{2}}\right) \\
& +\left(p-c_{h 1}+c_{h 2}\right) \sqrt{\sigma_{0}^{2}+\sigma_{2}^{2}} \Psi\left(\left(\theta_{1}-k_{2}\right) / \sqrt{\sigma_{0}^{2}+\sigma_{2}^{2}}\right) \\
& +\left(p-c_{h 1}+c_{h 2}\right) \theta_{1}+c_{h 1} k_{2}-c_{h 2} q_{1} \text {. }
\end{aligned}
$$

Proof: See appendix C.

We will show $E_{(1)}\left[\Pi\left(q_{1}\right)\right]_{\left(q_{1}, q_{2}\right) \in \Omega_{1}}$ and

$E_{(1)}\left[\Pi\left(q_{1}\right)\right]_{\left(q_{1}, q_{2}\right) \in \Omega_{2}}$ are both concave functions. Then we can search for optimal ordering quantity for each domain, expressed as $\left(q_{11}^{*}, q_{21}^{*}\right)$ and $\left(q_{12}^{*}, q_{22}^{*}\right)$ respec- tively.

Proposition 3. If $p+c_{s 1}+c_{h 2}>0$ and $p+c_{s 2}+c_{h 2}>0$ hold for $q_{1} \in[0, \infty)$, then $E_{(1)}\left[\Pi\left(q_{1}\right)\right]_{\left(q_{1}, q_{2}\right) \in \Omega_{1}}$ and $E_{(1)}\left[\Pi\left(q_{1}\right)\right]_{\left(q_{1}, q_{2}\right) \in \Omega_{2}}$ are concave functions of $q_{1}$. where

$$
\begin{gathered}
\frac{\partial^{2} E_{(1)}\left[\Pi\left(q_{1}\right)_{\left(q_{1}, q_{2}\right) \in \Omega_{1}}\right]}{\partial q_{1}^{2}}=\sum_{i=1}^{n} p_{i}\left(-\left(p+c_{s 1}+c_{h 2}\right) B_{4} \Phi\left(\frac{q_{1}-\sqrt{\sigma_{0}^{2}+\sigma_{2}^{2}} \Phi^{-1}\left(t_{1}\right)-B_{2}}{\sqrt{B_{1}}}\right)\right) \\
\frac{\partial^{2} E_{(1)}\left[\Pi\left(q_{1}\right)_{\left(q_{1}, q_{2}\right) \in \Omega_{2}}\right]}{\partial q_{1}^{2}}=\sum_{i=1}^{n} p_{i}\left(-\left(p+c_{s 2}+c_{h 2}\right) B_{4} \Phi\left(\frac{q_{1}-\sqrt{\sigma_{0}^{2}+\sigma_{2}^{2}} \Phi^{-1}\left(t_{2}\right)-B_{2}}{\sqrt{B_{1}}}\right)\right) \\
B_{1}=\frac{\sigma_{1}^{4}\left(\sigma_{2}^{2}+\sigma_{0}^{2}\right)}{\sigma_{1}^{4}+\left(\sigma_{1}^{4}+\sigma_{0}^{2}\right)\left(\sigma_{2}^{2}+\sigma_{0}^{2}\right)}, \\
B_{4}=\frac{\sqrt{\sigma_{1}^{2}+\sigma_{0}^{2}} \sqrt{B_{1}}}{\sqrt{2 \pi} \sigma_{1}^{2}\left(\sigma_{2}^{2}+\sigma_{0}^{2}\right)} e^{\left(B_{2}^{2}-B_{3}\right) / 2 B_{1}} .
\end{gathered}
$$$$
B_{2}=\frac{\sigma_{1}^{4} q_{1}+\theta_{1}\left(\sigma_{1}^{2}+\sigma_{0}^{2}\right)\left(\sigma_{2}^{2}+\sigma_{0}^{2}\right)}{\sigma_{1}^{4}+\left(\sigma_{1}^{2}+\sigma_{0}^{2}\right)\left(\sigma_{1}^{2}+\sigma_{0}^{2}\right)},
$$$$
B_{3}=\frac{\sigma_{1}^{4} q_{1}^{2}+\theta_{1}^{2}\left(\sigma_{1}^{2}+\sigma_{0}^{2}\right)\left(\sigma_{2}^{2}+\sigma_{0}^{2}\right)}{\sigma_{1}^{4}+\left(\sigma_{1}^{2}+\sigma_{0}^{2}\right)\left(\sigma_{2}^{2}+\sigma_{0}^{2}\right)},
$$ 
by the following procedure:

Step 1: At stage 1, we find $q_{11}^{*}, q_{12}^{*}$ such that

$$
\begin{aligned}
& \partial E_{(1)}\left[\Pi\left(q_{1}\right)_{\left(q_{1}, q_{2}\right) \in \Omega_{1}}\right] /\left.\partial q_{1}\right|_{q_{11}^{\prime}}=0 \\
& t_{1}=\left[p+c_{s 1} F_{x_{2}}\left((1+\gamma) \theta_{1}\right)-c^{(2)}\right] /\left(p+c_{h 2}+c_{s 1}\right), \\
& t_{2}=\left(p+c_{s 2}-c^{(2)}\right) /\left(p+c_{h 2}+c_{s 2}\right) \\
& q_{11}^{*}=\min \left[q_{11}^{\prime},(1+\gamma) \theta_{1}\right] \\
& q_{12}^{*}=q_{12}^{\prime} \\
& q_{21}^{*}=\left\{\begin{array}{l}
\max \left\{0, k_{2}+\sqrt{\sigma_{0}^{2}+\sigma_{2}^{2}} \Phi^{-1}\left(t_{1}\right)-q_{11}^{*}\right\} \\
\quad \text { if } \theta_{1} \leq k_{2}+\sqrt{\sigma_{0}^{2}+\sigma_{2}^{2}} \Phi^{-1}\left(t_{1}\right) \leq(1+\gamma) \theta_{1}, \\
\max \left\{0,\left\{(1+\gamma) \theta_{1}-q_{11}^{*}\right\}\right\} \\
\quad \text { if } k_{2}+\sqrt{\sigma_{0}^{2}+\sigma_{2}^{2}} \Phi^{-1}\left(t_{1}\right)>(1+\gamma) \theta_{1} \\
\max \left\{0,\left\{\theta_{1}-q_{11}^{*}\right\}\right\} \\
\quad \text { if } k_{2}+\sqrt{\sigma_{0}^{2}+\sigma_{2}^{2}} \Phi^{-1}\left(t_{1}\right)<\theta_{1}
\end{array},\right.
\end{aligned}
$$$$
\partial E_{(1)}\left[\Pi\left(q_{1}\right)_{\left(q_{1}, q_{2}\right) \in \Omega_{1}}\right] / \partial q_{1}=\sum_{i=1}^{n} p_{i}\left(\left(p+c_{s 1}-c^{(2)}\right) \Phi\left(\left[q_{1}-\sqrt{\sigma_{0}^{2}+\sigma_{2}^{2}} \Phi^{-1}\left(t_{1}\right)-\theta_{1}\right] /\left[\sqrt{\sigma_{1}^{4} /\left(\sigma_{0}^{2}+\sigma_{1}^{2}\right)}\right]\right)\right.
$$$$
\left.+\left(c^{(2)}-c^{(1)}\right)-\left(p+c_{s 1}+c_{h 2}\right) \times \int_{-\infty}^{q_{1}-\sqrt{d_{1}+d_{3}} \Phi^{-1}\left(t_{1}\right)} \Phi\left(\left(q_{1}-k_{2}\right) / \sqrt{\sigma_{0}^{2}+\sigma_{2}^{2}}\right) f_{K_{2}}\left(k_{2}\right) \mathrm{d} k_{2}\right)
$$$$
\partial E_{(1)}\left[\Pi\left(q_{1}\right)_{\left(q_{1}, q_{2}\right) \in \Omega_{2}}\right] / \partial q_{1}=\sum_{i=1}^{n} p_{i}\left(\left(p+c_{s 2}-c^{(2)}\right) \Phi\left(\left[q_{1}-\sqrt{\sigma_{0}^{2}+\sigma_{2}^{2}} \Phi^{-1}\left(t_{2}\right)-\theta_{1}\right] /\left[\sqrt{\sigma_{1}^{4} /\left(\sigma_{0}^{2}+\sigma_{1}^{2}\right)}\right]\right)\right.
$$$$
\left.+\left(c^{(2)}-c^{(1)}\right)-\left(p+c_{s 2}+c_{h 2}\right) \times \int_{-\infty}^{q_{1}-\sqrt{d_{1}+d_{3}} \Phi^{-1}\left(t_{2}\right)} \Phi\left(\left(q_{1}-k_{2}\right) / \sqrt{\sigma_{0}^{2}+\sigma_{2}^{2}}\right) f_{K_{2}}\left(k_{2}\right) \mathrm{d} k_{2}\right)
$$

and

$$
\partial E_{(1)}\left[\Pi\left(q_{1}\right)_{\left(q_{1}, q_{2}\right) \in \Omega_{2}}\right] /\left.\partial q_{1}\right|_{q_{12}^{\prime}}=0,
$$

where and

where

$$
\begin{aligned}
t_{1}=\left(p+c_{s 1} F_{2}\left((1+\gamma) \theta_{1}\right)-c^{(2)}\right) /\left(p+c_{h 2}+c_{s 1}\right) . \\
q_{22}^{*}=\left\{\begin{array}{c}
\max \left\{0,\left\{(1+\gamma) \theta_{1}-q_{12}^{*}\right\}\right\} \\
\quad \text { if } \theta_{1} \leq k_{2}+\sqrt{\sigma_{0}^{2}+\sigma_{2}^{2}} \Phi^{-1}\left(t_{2}\right) \leq(1+\gamma) \theta_{1} \\
\max \left\{0,\left\{k_{2}+\sqrt{\sigma_{0}^{2}+\sigma_{2}^{2}} \Phi^{-1}\left(t_{2}\right)-q_{12}^{*}\right\}\right\} \\
\quad \text { if } k_{2}+\sqrt{\sigma_{0}^{2}+\sigma_{2}^{2}} \Phi^{-1}\left(t_{2}\right)>(1+\gamma) \theta_{1}
\end{array}\right.
\end{aligned}
$$

where

$$
t_{2}=\left(p+c_{s 2}-c^{(2)}\right) /\left(p+c_{h 2}+c_{s 2}\right) .
$$

Step 3:

After determining optimal ordering quantity $\left(q_{11}^{*}, q_{21}^{*}\right)$ and $\left(q_{12}^{*}, q_{22}^{*}\right)$ for each domain, the optimal order quantity $q_{1}^{*}$ and $q_{2}^{*}$ can be derived as follows:

$$
\begin{gathered}
q_{1}^{*}=\operatorname{Arg}_{\left(q_{1}\right)} \max \left\{E_{(1)}\left[\Pi\left(q_{1}\right)_{\left(q_{1}\right) \in \Omega_{1}} \mid q_{1}=q_{11}^{*}\right],\right. \\
\left.E_{(1)}\left[\Pi\left(q_{1}\right)_{\left(q_{1}\right) \in \Omega_{2}} \mid q_{1}=q_{12}^{*}\right]\right\} \\
q_{2}^{*}=\left\{\begin{array}{l}
q_{21}^{*}, \text { if } q_{1}^{*}=q_{11}^{*} \\
q_{22}^{*}, \text { if } q_{1}^{*}=q_{12}^{*}
\end{array}\right.
\end{gathered}
$$

In next section we will demonstrate the proposed procedure with some given numerical examples and the sensitivity analysis.

\section{Computational Study}

\subsection{Numerical Examples}

Three examples are presented to demonstrate the proposed solution procedure. The relevant parameters are shown in Table 1 . The optimum $q_{1}^{*}, q_{2}^{*}$ and the corresponding expected profit for each example are also shown in Table 1.

Example 1. Suppose the buyer demand follows a normal distribution with the standard deviation terms $\sigma_{0}=3$, $\sigma_{1}=5$, and buyer's minimum-commitment quantity $\left(\theta_{1}\right)$ is 30 and $\gamma$ is 0.1 , that is, shortage compensation range is $(30,33)$. The demand observation at the second stage $\left(\theta_{2}\right)$ is 33 , and the other relevant parameters are given as follows: product unit selling price $p$ is 100 , the unit cost of key component at stage $1, c^{(1)}=\$ 30$, there is a $70 \%$ 
Table 1. Examples.

\begin{tabular}{|c|c|c|c|}
\hline & Example 1 & Example 2 & Example 3 \\
\hline$\sigma_{0}$ & 3 & 3 & 3 \\
\hline$\sigma_{1}$ & 5 & 5 & 5 \\
\hline$p$ & 100 & 100 & 100 \\
\hline$C_{1}$ & 30 & 30 & 30 \\
\hline$c_{1}^{(2)}\left(p_{1}=0.7\right)$ & 40 & 40 & 40 \\
\hline$c_{2}^{(2)}\left(p_{2}=0.3\right)$ & 20 & 20 & 20 \\
\hline$\theta_{1}$ & 30 & 30 & 30 \\
\hline$\gamma$ & 0.1 & 0.4 & 0.1 \\
\hline$\theta_{2}$ & 33 & 33 & 38 \\
\hline$c_{h 1}$ & 10 & 10 & 10 \\
\hline$c_{h 2}$ & 15 & 15 & 15 \\
\hline$c_{s 1}$ & 15 & 15 & 15 \\
\hline$c_{\mathrm{s} 2}$ & 10 & 10 & 10 \\
\hline Fitted domain & $\Omega_{2}$ & $\Omega_{1}$ & $\Omega_{2}$ \\
\hline \multirow{5}{*}{$\begin{array}{c}\text { Optimal Solution } \\
q_{1}^{*}, q_{2}^{*}\end{array}$} & $q_{1}^{*}=27.1216$ & $q_{1}^{*}=27.2491$ & $q_{1}^{*}=27.1216$ \\
\hline & $q_{2}^{*}=5.8784$ & $q_{2}^{*}=5.7159$ & $q_{2}^{*}=9.3574$ \\
\hline & $\left(c^{(2)}=40\right)$ & $\left(c^{(2)}=40\right)$ & $\left(c^{(2)}=40\right)$ \\
\hline & $q_{2}^{*}=7.3876$ & $q_{2}^{*}=7.3787$ & $q_{2}^{*}=11.0641$ \\
\hline & $\left(c^{(2)}=20\right)$ & $\left(c^{(2)}=20\right)$ & $\left(c^{(2)}=20\right)$ \\
\hline Optimal Profit & 2084.91. & 2079.22 & 2220.19 \\
\hline
\end{tabular}

chance that the ordering cost at stage 2 will 40 and a $30 \%$ that the ordering cost at stage 2 is $20\left(c_{1}^{(2)}=40\right.$, $\left.p_{1}=0.7, c_{2}^{(2)}=20, \quad p_{2}=0.3\right)$, per unit holding cost of buyer responsible inventory $\left(c_{h 1}\right)=\$ 10$, per unit holding cost of manufacturer responsible inventory $\left(c_{h 2}\right)=\$ 15$, per unit shortage compensation cost $\left(c_{s 1}\right)=\$ 15$ and per unit general shortage cost $\left(c_{s 2}\right)=\$ 10$.

With the proposed solution procedure, in step 1 we find that $q_{11}^{\prime}=27.3127$ and $q_{12}^{\prime}=27.1216$, then

$$
q_{11}^{*}=\min \left[27.3127,(1+\gamma) \theta_{1}=33\right]=27.3127
$$

and $q_{12}^{*}=27.1216$. In step 2, if $c_{1}^{(2)}=40$ at stage 2,

$$
\begin{aligned}
\left(\theta_{1}=30\right) & <\left(k_{2}+\sqrt{\sigma_{0}^{2}+\sigma_{2}^{2}} \Phi^{-1}\left(t_{1}\right)=32.4876\right) \\
& <\left((1+\gamma) \theta_{1}=33\right)
\end{aligned}
$$

and

$$
\begin{aligned}
(\theta=30) & <\left(k_{2}+\sqrt{\sigma_{0}^{2}+\sigma_{2}^{2}} \Phi^{-1}\left(t_{2}\right)=32.8025\right), \\
& <\left((1+\gamma) \theta_{1}=33\right)
\end{aligned}
$$

then

$$
q_{21}^{*}=\max \{0,(32.4876-27.3127)\}=5.1749
$$

and

$$
q_{22}^{*}=\max \{0,\{33-27.1216\}\}=5.8784 .
$$

If $c_{2}^{(2)}=20$ at stage 2 ,

$$
\left((1+\gamma) \theta_{1}=33\right)<\left(k_{2}+\sqrt{\sigma_{0}^{2}+\sigma_{2}^{2}} \Phi^{-1}\left(t_{1}\right)=34.0793\right)
$$

and

$$
\left((1+\gamma) \theta_{1}=33\right)<\left(k_{2}+\sqrt{\sigma_{0}^{2}+\sigma_{2}^{2}} \Phi^{-1}\left(t_{2}\right)=34.5092\right),
$$

then

$$
q_{21}^{*}=\max \{0,\{33-27.3127\}\}=5.6873
$$

and

$$
q_{22}^{*}=\max \{0,\{34.5092-27.1216\}\}=7.3876 .
$$

In step 3, we get

$$
\begin{aligned}
q_{1}^{*}= & \operatorname{Arg}_{\left(q_{1}\right)} \max \left\{E_{(1)}\left[\Pi\left(q_{1}\right)_{\left(q_{1}\right) \in \Omega_{1}} \mid q_{1}=q_{11}^{*}\right],\right. \\
& \left.E_{(1)}\left[\Pi\left(q_{1}\right)_{\left(q_{1}\right) \in \Omega_{2}} \mid q_{1}=q_{12}^{*}\right]\right\} \\
= & \operatorname{Arg}_{\left(q_{1}\right)} \max \{2081.85,2084.91\}=27.1216,
\end{aligned}
$$

$q_{2}^{*}=5.8784\left(\right.$ if $\left.c_{1}^{(2)}=40\right)$ or $q_{2}^{*}=7.3876$ (if $c_{2}^{(2)}=20$ ) and optimal expected profit is 2084.91 .

Example 2. In example 1 the value of $\gamma$ is changed from 0.1 to 0.4 while other parameters remain unchanged. With the proposed solution procedure, in step 1 we find that $q_{11}^{\prime}=27.2491$ and $q_{12}^{\prime}=27.1216$, then

$$
q_{11}^{*}=\min \left[27.2491,(1+\gamma) \theta_{1}=42\right]=27.2491
$$

and $q_{12}^{\prime}=27.1216$. In step 2, if $c_{1}^{(2)}=40$ at stage 2,

$$
\begin{aligned}
\left(\theta_{1}=30\right) & <\left(k_{2}+\sqrt{\sigma_{0}^{2}+\sigma_{2}^{2}} \Phi^{-1}\left(t_{1}\right)=32.965\right) \\
& <\left((1+\gamma) \theta_{1}=42\right)
\end{aligned}
$$

and

$$
\begin{aligned}
(\theta=30) & <\left(k_{2}+\sqrt{\sigma_{0}^{2}+\sigma_{2}^{2}} \Phi^{-1}\left(t_{2}\right)=32.8025\right), \\
& <\left((1+\gamma) \theta_{1}=42\right)
\end{aligned}
$$

then

$$
q_{21}^{*}=\max \{0,(32.965-27.2491)\}=5.7159
$$

and

$$
q_{22}^{*}=\max \{0,\{42-27.1216\}\}=14.8784 .
$$

If $c_{2}^{(2)}=20$ at stage 2 , 


$$
\begin{aligned}
\left(\theta_{1}=30\right) & <\left(k_{2}+\sqrt{\sigma_{0}^{2}+\sigma_{2}^{2}} \Phi^{-1}\left(t_{1}\right)=34.6278\right) \\
& <\left((1+\gamma) \theta_{1}=42\right)
\end{aligned}
$$

and

$$
\left(k_{2}+\sqrt{\sigma_{0}^{2}+\sigma_{2}^{2}} \Phi^{-1}\left(t_{2}\right)=34.5092\right)<\left((1+\gamma) \theta_{1}=42\right),
$$

then

$$
q_{21}^{*}=\max \{0,\{34.6278-27.2491\}\}=7.3787
$$

and

$$
q_{22}^{*}=\max \{0,\{42-27.1216\}\}=14.8784 .
$$

In step 3, we get

$$
\begin{aligned}
q_{1}^{*}= & \operatorname{Arg}_{\left(q_{1}\right)} \max \left\{E_{(1)}\left[\Pi\left(q_{1}\right)_{\left(q_{1}\right) \in \Omega_{1}} \mid q_{1}=q_{11}^{*}\right],\right. \\
& \left.E_{(1)}\left[\Pi\left(q_{1}\right)_{\left(q_{1}\right) \in \Omega_{2}} \mid q_{1}=q_{12}^{*}\right]\right\} \\
= & \operatorname{Arg}_{\left(q_{1}\right)} \max \{2079.22,1846.81\}=27.2491,
\end{aligned}
$$

$q_{2}^{*}=5.7159$ (if $c_{1}^{(2)}=40$ ) or $q_{2}^{*}=7.3787$ (if $c_{2}^{(2)}=20$ ) and optimal expected profit is 2079.22 .

Example 3. In example 1 the value of $\theta_{2}$ is changed from 33 to 38 while other parameters remain unchanged. With the proposed solution procedure, in step 1 we find that $q_{11}^{\prime}=27.4702$ and $q_{12}^{\prime}=27.1216$, then

$$
q_{11}^{*}=\min \left[27.4702,(1+\gamma) \theta_{1}=42\right]=27.4702
$$

and $q_{12}^{\prime}=27.1216$. In step 2, if $c_{1}^{(2)}=40$ at stage 2,

$$
\left((1+\gamma) \theta_{1}=33\right)<\left(k_{2}+\sqrt{\sigma_{0}^{2}+\sigma_{2}^{2}} \Phi^{-1}\left(t_{1}\right)=35.7675\right)
$$

and

$$
\left((1+\gamma) \theta_{1}=33\right)<\left(k_{2}+\sqrt{\sigma_{0}^{2}+\sigma_{2}^{2}} \Phi^{-1}\left(t_{2}\right)=36.479\right),
$$

then

$$
q_{21}^{*}=\max \{0,(33-27.4702)\}=5.5298
$$

and

$$
q_{22}^{*}=\max \{0,\{36.479-27.1216\}\}=9.3574 .
$$

If $c_{2}^{(2)}=20$ at stage 2,

$$
\left((1+\gamma) \theta_{1}=33\right)<\left(k_{2}+\sqrt{\sigma_{0}^{2}+\sigma_{2}^{2}} \Phi^{-1}\left(t_{1}\right)=37.3228\right)
$$

and

$$
\left((1+\gamma) \theta_{1}=33\right)<\left(k_{2}+\sqrt{\sigma_{0}^{2}+\sigma_{2}^{2}} \Phi^{-1}\left(t_{2}\right)=38.1857\right),
$$

then

$$
q_{21}^{*}=\max \{0,\{33-27.4702\}\}=5.5298
$$

and

$$
q_{22}^{*}=\max \{0,\{38.1857-27.1216\}\}=11.0641 .
$$

In step 3, we get

$$
\begin{aligned}
q_{1}^{*}= & \operatorname{Arg}_{\left(q_{1}\right)} \max \left\{E_{(1)}\left[\Pi\left(q_{1}\right)_{\left(q_{1}\right) \in \Omega_{1} \mid} \mid q_{1}=q_{11}^{*}\right],\right. \\
& \left.E_{(1)}\left[\Pi\left(q_{1}\right)_{\left(q_{1}\right) \in \Omega_{2}} \mid q_{1}=q_{12}^{*}\right]\right\} \\
= & \operatorname{Arg}_{\left(q_{1}\right)} \max \{2131.75,2220.19\}=27.1216,
\end{aligned}
$$

$q_{2}^{*}=9.3574$ (if $c_{1}^{(2)}=40$ ) or 11.0641 (if $c_{2}^{(2)}=20$ ) and optimal expected profit is 2220.19 .

\subsection{Sensitivity Analysis}

$\theta_{2}$ is an observation of $N\left(\theta_{1}, \sigma_{0}^{2}+\sigma_{1}^{2}\right)$ at stage 2. At stage 1 we don't know what value of $\theta_{2}$ will be observed. With Monte Carlo method we randomly generate 100 values of $\theta_{2}$ from $N\left(\theta_{1}, \sigma_{0}^{2}+\sigma_{1}^{2}\right)$, denoted as $\theta_{2}^{(i)}$, $i=1, \cdots, 100$. With the proposed solution procedure, we can find $q_{1}^{*(i)}$ with respect to $\theta_{2}^{(i)}$. Then we evaluate the average expected profit value for using $q_{1}^{*(i)}$ in 100 values of $\theta_{2}^{(i)}$. The steps of Monte Carlo method are stated as follows:

Step 1: Randomly generate $\theta_{2}^{(i)}, i=1, \cdots, 100$ from $N\left(\theta_{1}, \sigma_{0}^{2}+\sigma_{1}^{2}\right)$.

Step 2: With each $\theta_{2}^{(i)}$ we can find $q_{1}^{*(i)}$ by Proposition $5, i=1, \cdots, 100$ respectively.

Step 3: Compute the expected profit values for using $q_{1}^{*(i)}$ in each $\theta_{2}^{(i)} i=1, \cdots, 100$, denoted as $\Pi^{(1)}\left(q_{1}^{*(i)}\right), \cdots$, $\Pi^{(100)}\left(q_{1}^{*(i)}\right)$ (excluding $\theta_{2}$, parameters are fixed in $\left.\Pi^{(i)}\left(q_{1}^{*(i)}\right), i=1, \cdots, 100\right)$. Let

$$
\begin{gathered}
\prod_{(i)}^{*}=\left[\Pi^{(1)}\left(q_{1}^{*(i)}\right)+\Pi^{(2)}\left(q_{1}^{*(i)}\right)+\cdots+\Pi^{(100)}\left(q_{1}^{*(i)}\right)\right] / 100, \\
i=1, \cdots, 100 .
\end{gathered}
$$

Step 4: optimal profit and optimal order quantity $q_{1}^{*}$ can be derived as follows:

$$
q_{1}^{*}=\operatorname{Arg}_{\left(q_{1}^{*(i)}\right)} \max \left\{\prod_{(1)}^{*}, \prod_{(2)}^{*}, \cdots, \prod_{(100)}^{*}\right\} .
$$

The relationships among optimal expected profit, shortage compensation range coefficient $(\gamma)$, and buyer's minimum-commitment quantity $\left(\theta_{1}\right)$ are shown in Table 1 with the related parameters given in the illustrating example. And the relationships among optimal expected profit, shortage compensation range coefficient $(\gamma)$ and shortage compensation $\operatorname{cost} c_{s 1}$, are shown in Table 2 and Table 3.

In Table 2 we find that: 1) given $\theta_{1}$, we define the optimal expected profit turning point of decreasing as 
Table 2. Relationships among optimal expected profit, shortage compensation coefficient and buyer's minimum-commitment quantity.

\begin{tabular}{|c|c|c|c|c|c|}
\hline 0 & 20 & 25 & 30 & 35 & 40 \\
\hline 0 & 1836.02 & 2074.59 & 2345.81 & 2653.83 & 2990.32 \\
\hline 0.1 & 1836.02 & 2074.59 & 2345.81 & 2653.83 & 2990.32 \\
\hline 0.2 & 1836.02 & 2074.59 & 2345.81 & 2653.83 & 2990.32 \\
\hline 0.3 & 1836.02 & 2074.59 & 2345.81 & 2653.83 & 2990.32 \\
\hline 0.4 & 1836.02 & 2074.59 & 2345.81 & 2648.21 & 2985.97 \\
\hline 0.5 & 1836.02 & 2067.19 & 2337.45 & 2645.42 & 2982.73 \\
\hline 0.6 & 1832.17 & 2064.98 & 2335.56 & 2643.19 & 2980.85 \\
\hline 0.7 & 1829.75 & 2064.86 & 2335.53 & 2643.11 & 2980.44 \\
\hline 0.8 & 1829.53 & 2064.67 & 2335.47 & 2643.1 & 2980.41 \\
\hline 0.9 & 1829.49 & 2064.65 & 2335.47 & 2643.1 & 2980.39 \\
\hline 1 & 1829.48 & 2064.65 & 2335.45 & 2643.1 & 2980.39 \\
\hline
\end{tabular}

Table 3. Relationships among optimal expected profit, shortage compensation coefficient and unit shortage compensation cost $\left(c_{s 1}\right)$ (given $\left.\theta_{1}=30\right)$.

\begin{tabular}{|c|c|c|c|c|c|}
\hline & 15 & 30 & 45 & 60 & 75 \\
\hline 0 & 2345.81 & 2345.81 & 2345.81 & 2345.81 & 2345.81 \\
\hline 0.1 & 2345.81 & 2345.81 & 2345.81 & 2345.81 & 2345.81 \\
\hline 0.2 & 2345.81 & 2345.81 & 2345.81 & 2345.81 & 2345.81 \\
\hline 0.3 & 2345.81 & 2345.81 & 2345.81 & 2345.81 & 2345.81 \\
\hline 0.4 & 2345.81 & 2345.81 & 2345.81 & 2345.81 & 2345.81 \\
\hline 0.5 & 2337.45 & 2321.75 & 2312.85 & 2299.03 & 2270.26 \\
\hline 0.6 & 2335.56 & 2315.03 & 2301.06 & 2283.1 & 2264.03 \\
\hline 0.7 & 2335.53 & 2303.37 & 2290.98 & 2270.67 & 2243.73 \\
\hline 0.8 & 2335.47 & 2302.57 & 2287.84 & 2266.5 & 2229.04 \\
\hline 0.9 & 2335.47 & 2302.48 & 2286.71 & 2260.49 & 2228.85 \\
\hline 1 & 2335.45 & 2302.47 & 2286.7 & 2260.49 & 2228.84 \\
\hline
\end{tabular}

$\gamma_{\theta_{1}}$, that is, when $\gamma<\gamma_{\theta_{1}}$, the optimal expected profit is kept unchanged, but when $\gamma>\gamma_{\theta_{1}}$, the optimal expected profit decreases accordingly, i.e., $\gamma_{20}=0.5, \gamma_{25}=\gamma_{30}=$ $0.4, \gamma_{35}=\gamma_{40}=0.3$. The larger $\theta_{1}$ is, the smaller $\gamma_{\theta_{1}}$ is. 2) The marginal optimal expected profit is increased as $\theta_{1}$ increases. In Table 3, Table 4 and Figure 3 with a given $\theta_{1}$ we find that when $\gamma<\gamma_{\theta_{1}}\left(\gamma_{30}=0.4, \gamma_{40}=\right.$ 0.3 ), the optimal total order quantity $q_{1}^{*}+q_{2}^{*}$ is over $(1+\gamma) \theta_{1}$ which causes the shortage compensation cost can not be occurred. Hence, if $\gamma<\gamma_{40}$ then the manufacturer can give the buyer a larger shortage compensation value of $c_{s 1}$ and take buyer's increasing the value of $\theta_{1}$. For example, if the original shortage compensation coefficient $\gamma=0.2$ and $\theta_{1}=30$, then we find $\gamma_{30}=0.4$, the manufacturer can give $\gamma=0.3$ and an arbitrarily large value of compensation cost $C_{\mathrm{s} 1}$ to the buyer and take the buyer increasing the value of $\theta_{1}$ from 30 to 40 , then the optimal expected profit in the case of $\gamma=0.3$ and $\theta_{1}=40$ will be larger than it in the case of $\gamma=0.2$ and $\theta_{1}=30$.

Base on the above description, the management insights observed from Table 2 to Table 4 can be concluded as follows:

1) The more the buyer's minimum-commitment quantity $\theta_{1}$ is, the more the expected profit of manufacturer is.

2) There are some ways to induce the buyer to increase the value of $\theta_{1}$ :

a) From Table 2, we know the upper bound of $\gamma\left(\gamma_{\theta_{1}}\right)$ which the manufacturer can give to the buyer to attract the buyer increasing the minimum commitment value of $\theta_{1}$ without losing the expected profit.

b) If the optimal total order quantity $q_{1}^{*}+q_{2}^{*}$ is larger than $(1+\gamma) \theta_{1}$, then the manufacturer can give attractive values of shortage compensation cost $c_{s 1}$ (Tables 3 and 4) and shortage compensation coefficient $(\gamma)$ (Table 2 to Table 4 to take buyer increasing the value of $\theta_{1}$.

The manufacturer can provide alternatives for the buyer as Table 5 to induce the buyer to increase the value of $\theta_{1}$.

Table 4. Relationships among optimal expected profit, shortage compensation coefficient and unit shortage compensation cost $\left(c_{s 1}\right)$ (given $\theta_{1}=40$ ).

\begin{tabular}{|c|c|c|c|c|c|}
\hline 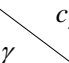 & 15 & 30 & 45 & 60 & 75 \\
\hline 0 & 2990.32 & 2990.32 & 2990.32 & 2990.32 & 2990.32 \\
\hline 0.1 & 2990.32 & 2990.32 & 2990.32 & 2990.32 & 2990.32 \\
\hline 0.2 & 2990.32 & 2990.32 & 2990.32 & 2990.32 & 2990.32 \\
\hline 0.3 & 2990.32 & 2990.32 & 2990.32 & 2990.32 & 2990.32 \\
\hline 0.4 & 2985.97 & 2963.11 & 2940.85 & 2934.03 & 2930.33 \\
\hline 0.5 & 2982.73 & 2956.39 & 2932.06 & 2909.27 & 2887.73 \\
\hline 0.6 & 2980.85 & 2952.73 & 2926.98 & 2903.1 & 2880.74 \\
\hline 0.7 & 2980.44 & 2951.93 & 2925.84 & 2901.67 & 2879.04 \\
\hline 0.8 & 2980.41 & 2951.84 & 2925.71 & 2901.5 & 2878.85 \\
\hline 0.9 & 2980.39 & 2951.83 & 2925.7 & 2901.49 & 2878.84 \\
\hline 1 & 2980.39 & 2951.83 & 2925.7 & 2901.49 & 2878.84 \\
\hline
\end{tabular}




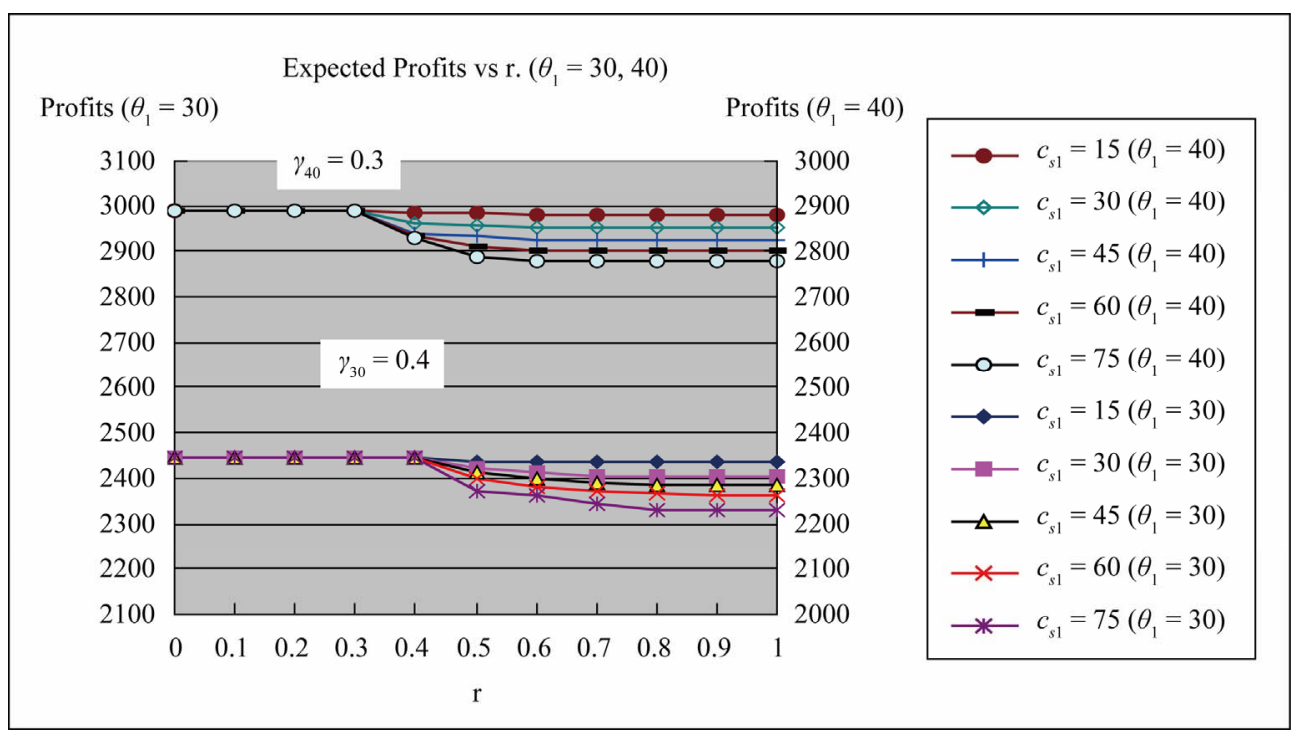

Figure 3. Relationships among the expected profit, shortage compensation cost $\left(c_{s 1}\right)$, shortage compensation coefficient $(\gamma)$ and unit shortage compensation cost $\left(c_{s 1}\right)$.

Table 5. Alternatives for the buyer.

\begin{tabular}{cccc}
\hline Alternative & $\begin{array}{c}\text { Buyer's } \\
\text { minimum-commitment } \\
\text { quantity } \theta_{1}\end{array}$ & $\begin{array}{c}\text { Shortage } \\
\text { compensation } \\
\text { coefficient }\end{array}$ & $\begin{array}{c}\text { Shortage } \\
\text { compensation } \\
\text { cost } c_{s 1}\end{array}$ \\
\hline 1 & $\theta_{1}=20$ & $\gamma=0.1$ & $c_{s 1}=15$ \\
2 & $\theta_{1}=30$ & $\gamma=0.2$ & $c_{s 1}=30$ \\
3 & $\theta_{1}=40$ & $\gamma=0.3$ & $c_{s 1}=45$ \\
\hline
\end{tabular}

\section{Conclusions}

This paper proposes a two-stage dynamic optimization model for an ODM CMOS camera module manufacturer to determine its optimal order quantities to maximize optimal expected profit based on buyer's minimumcommitment quantity contract and shortage compensation policy. The manufacturer can update the distribution of buyer's demand by collecting the market information, and this situation is common and realistic for entrepreneurs in industry. In this paper, two kinds of inventories and shortage costs that are taken into consideration, the conditions for the two-stage optimal order quantities are derived, and the solution procedure is proposed. Numerical examples are to be illustrated and some management insights are provided. The upper bound of $\gamma$ $\left(\gamma_{\theta_{1}}\right)$ which the manufacturer can give to the buyer to attract the buyer increasing the minimum commitment value of $\theta_{1}$ without losing the expected profit, the manufacturer can use the upper bound of $\gamma\left(\gamma_{\theta_{1}}\right)$ to redefine an attractive values of shortage compensation coefficient $(\gamma)$ and shortage compensation cost $\left(c_{s 1}\right)$ to induce the buyer to increase the value of $\theta_{1}$ that can im- prove expected profit of the manufacturer. It would be of interest to extend the model to allow for the manufacturer having third or above order opportunity before the buyer's real demand occurred.

\section{References}

[1] R. Anupindi and Y. Bassok, "Supply Contracts with Quantity Commitments and Stochastic Demand," In: S. Tayur, R. Ganeshan and M. Magazine, Eds., Quantitative Models for Supply Chain Management, Kluwer Academic Publishers, Boston, 1999.

[2] S. Sethi, H. Yan and H. Zhang, "Inventory and Supply Chain Management with Forecast Updates," Kluwer Academic Publishers, Boston, 2005.

[3] E. J. Durango-Cohen and C. A. Yano, "Supplier Commitment and Production Decisions under a ForecastCommitment Contract," Management Science, Vol. 52, No. 1, 2006, pp. 54-67. doi:10.1287/mnsc.1050.0471

[4] Y. Bassok and R. Anupindi, "Analysis of Supply Contracts with Total Minimum Commitment," IIE Transactions, Vol. 29, No. 5, 1997, pp. 373-381. doi: $10.1080 / 07408179708966342$

[5] R. Anupindi and Y. Bassok, "Approximations for Multiproduct Contracts with Stochastic Demands and Business Volume Discounts: Single Supplier Case," IIE Transactions, Vol. 30, No. 8, 1998, pp. 723-734. doi: $10.1080 / 07408179808966518$

[6] S. Sethi and G. Sorger, "A Theory of Rolling Horizon Decision Making," Annals of Operations Research, Vol. 29, No. 1-4, 1991, pp. 387-416. doi:10.1007/BF02283607

[7] Y. Bassok and R. Anupindi, "Analysis of Supply Contracts with Commitments and Flexibility," Naval Research Logistics, Vol. 55, No. 5, 2008, pp. 459-477. 
doi:10.1002/nav.20300

[8] Z. Lian and A. Deshmukh, "Analysis of Supply Contracts with Quantity Flexibility," European Journal of Operational Research, Vol. 196, No. 2, 2009, pp. 526-533. doi:10.1016/j.ejor.2008.02.043

[9] G. Gallego and O. Ozer, "Integrating Replenishment Decisions with Advance Demand Information," Management Science, Vol. 47, No. 10, 2001, pp. 1344-1360. doi:10.1287/mnsc.47.10.1344.10261

[10] Y. Aviv, "A Time-Series Framework for Supply-Chain Inventory Management," Operations Research, Vol. 51, No. 2, 2003, pp. 210-227. doi:10.1287/opre.51.2.210.12780

[11] A. Dvoretzky, J. Kiefer and J. Wolfowitz, "The Inventory Problem: II. Case of Unknown Distribution of Demand," Econometrica, Vol. 20, No. 3, 1952, pp. 450-466.

[12] H. Scarf, "Bayes Solution of the Statistical Inventory Problem," Annals of Mathematical Statistics, Vol. 30, No. 2, 1959, pp. 490-508. doi:10.1214/aoms/1177706264

[13] H. Scarf, "Some Remarks on Bayes Solutions to the Inventory Problem," Naval Research Logistics, Vol. 7, No. 4, 1960, pp. 591-596. doi:10.1002/nav.3800070428

[14] M. A. Lariviere and E. L. Porteus, "Stalking Information: Bayesian Inventory Management with Unobserved Lost Sales," Management Science, Vol. 45, No. 3, 1999, pp. 346-363. doi: 10.1287/mnsc.45.3.346

[15] G. D. Eppen and A. V. Iyer, "Improved Fashion Buying with Bayesian Updates," Operations Research, Vol. 45, No. 6, 1997, pp. 805-819.

[16] J. Wu, "Quantity Flexibility Contracts under Bayesian Updating," Computers \& Operations Research, Vol. 32, No. 5, 2005, pp. 1267-1288.

[17] A. V. Iyer and M. E. Bergen, "Quick Response in Manufacturer-Retailer Channels," Management Science, Vol. 43, No. 4, 1997, pp. 559-570.

[18] T. M. Choi, D. Li and H. Yan, "Optimal Two-Stage Ordering Policy with Bayesian Information Updating," Journal of the Operation Research Society, Vol. 54, No. 8, 2003, pp. 846-859.

[19] O. Johnson and H. Thompson, "Optimality of Myopic
Inventory Policies for Certain Dependent Demand Processes," Management Science, Vol. 21, No. 11, 1975, pp. 1303-1307. doi: 10.1287/mnsc.21.11.1303

[20] W. H. Hausmann, "Sequential Decision Problems: A Model to Exploit Existing Forecast," Management Science, Vol. 16, No. 2, 1969, pp. B93-B111. doi:10.1287/mnsc.16.2.B93

[21] D. Heath and P. Jackson, "Modeling the Evolution of Demand Forecast with Application to Safety Stock Analysis in Production/Distribution Systems," IIE Transactions, Vol. 26. No. 3, 1994, pp. 17-30. doi:10.1080/07408179408966604

[22] Y. Wang and B. Tomlin, "To Wait or Not to Wait: Optimal Ordering under Lead Time Uncertainty and Forecast Updating," Naval Research Logistics, Vol. 56, No. 8, 2009, pp. 766-779. doi:10.1002/nav.20381

[23] H. Gurnani and C. S. Tang, "Optimal Ordering Decision with Uncertain Cost and Demand Forecast Updating," Management Science, Vol. 45, No. 10, 1999, pp. 14561462. doi: $10.1287 / \mathrm{mnsc} .45 .10 .1456$

[24] K. L. Donohue, "Efficient Supply Contract for Fashion Goods with Forecast Updating and Two Production Modes," Management Science, Vol. 46, No. 11, 2000, pp. 13971411. doi:10.1287/mnsc.46.11.1397.12088

[25] H. Y. Huang, S. Sethi and H. Yan, "Purchase Contract Management with Demand Forecast Updates," IIE Transactions, Vol. 37, No. 8, 2005, pp. 775-785. doi:10.1080/07408170590961157

[26] H. Chen, J. Chen and Y. Chen, "A Coordination Mechanism for a Supply Chain with Demand Information Updating," International Journal of Production Economics, Vol. 103, No. 1, 2006, pp. 347-361. doi:10.1016/j.ijpe.2005.09.002

[27] H. Yan, K. Liu and A. Hsu, "Order Quantity in Dual Supply Mode with Updating Forecasts," Production and Operations Management, Vol. 12, No. 1, 2003, pp. 30-45. doi:10.1111/j.1937-5956.2003.tb00196.x

[28] S. Sethi, H. Yan and H. Zhang, "Quantity Flexibility Contracts: Optimal Decisions with Information Updates," Decision Sciences, Vol. 35, No. 4, 2004, pp. 691-712. doi:10.1111/j.1540-5915.2004.02873.x 


\section{Appendix A}

Proof of Proposition 1:

$$
\begin{aligned}
& \mathrm{d} E_{(2)}\left[\Pi\left(q_{2} \mid q_{1}\right)\right]_{\left(q_{1}, q_{2}\right) \in \Omega_{1}} / \mathrm{d} q_{2} \\
& =\int_{q_{1}+q_{2}}^{\infty} p f_{2}(x) \mathrm{d} x-c_{h 2} \int_{-\infty}^{\theta_{1}} f_{2}(x) \mathrm{d} x \\
& \quad-c_{h 2} \int_{\theta_{1}}^{q_{1}+q_{2}} f_{2}(x) \mathrm{d} x-c_{s 1} \int_{q_{1}+q_{2}}^{(1+\gamma) \theta_{1}} f_{2}(x) \mathrm{d} x-c^{(2)}
\end{aligned}
$$

After rearranging the above equation, we have

$$
\begin{aligned}
& \mathrm{d} E_{(2)}\left[\Pi\left(q_{2} \mid q_{1}\right)\right]_{\left(q_{1}, q_{2}\right) \in \Omega_{1}} / \mathrm{d} q_{2} \\
& =-\left(p+c_{h 2}+c_{s 1}\right) F_{2}\left(q_{1}+q_{2}\right)+p+c_{s 1} F_{2}\left((1+\gamma) \theta_{1}\right)-c^{(2)}
\end{aligned}
$$$$
\text { and } \mathrm{d}^{2} E_{(2)}\left[\Pi\left(q_{2} \mid q_{1}\right)\right]_{\left(q_{1}, q_{2}\right) \in \Omega_{1}} / \mathrm{d} q_{2}^{2}
$$$$
=-\left(p+c_{h 2}+c_{s 1}\right) f_{2}\left(q_{1}+q_{2}\right) .
$$

If $p+c_{s 1}+c_{h 2}>0$ hold for $q_{2} \in[0, \infty)$, then

$$
\mathrm{d}^{2} E_{(2)}\left[\Pi\left(q_{2} \mid q_{1}\right)\right]_{\left(q_{1}, q_{2}\right) \in \Omega_{1}} / \mathrm{d} q_{2}^{2} \leq 0 .
$$

Therefore $E_{(2)}\left[\Pi\left(q_{2} \mid q_{1}\right)\right]_{\left(q_{1}, q_{2}\right) \in \Omega_{1}}$ is concave for $q_{2} \in[0, \infty)$. The proof of $E_{(2)}\left[\Pi\left(q_{2} \mid q_{1}\right)\right]_{\left(q_{1}, q_{2}\right) \in \Omega 2}$ is the same as $E_{(2)}\left[\Pi\left(q_{2} \mid q_{1}\right)\right]_{\left(q_{1}, q_{2}\right) \in \Omega_{1}}$.

\section{Appendix B}

Proof of Proposition 2: For $\Omega_{1}$, let

$$
\begin{aligned}
& \partial E_{(2)}\left[\Pi\left(q_{2} \mid q_{1}\right)\right]_{\left(q_{1}, q_{2}\right) \in \Omega 2} / \partial q_{2} \\
& =-\left(p+c_{h 2}+c_{s 1}\right) F_{2}\left(q_{1}+q_{2}\right)+p \\
& +c_{s 1} F_{2}\left((1+\gamma) \theta_{1}\right)-c^{(2)}=0
\end{aligned}
$$

If

$$
\begin{gathered}
\theta_{1} \leq k_{2}+\sqrt{\sigma_{0}^{2}+\sigma_{2}^{2}} \Phi^{-1}\left(t_{1}\right) \leq(1+\gamma) \theta_{1}, \\
q_{21}^{*}=\max \left\{0, k_{2}+\sqrt{\sigma_{0}^{2}+\sigma_{2}^{2}} \Phi^{-1}\left(t_{1}\right)-q_{1}\right\},
\end{gathered}
$$

where $t_{1}=\left(p+c_{s 1} F_{2}\left((1+\gamma) \theta_{1}\right)-c^{(2)}\right) /\left(p+c_{h 2}+c_{s 1}\right)$.

If $k_{2}+\sqrt{\sigma_{0}^{2}+\sigma_{2}^{2}} \Phi^{-1}\left(t_{1}\right)>(1+\gamma) \theta_{1}$,

we take the upper bound of $\Omega_{1}\left((1+\gamma) \theta_{1}\right)$ instead of $\left(k_{2}+\sqrt{\sigma_{0}^{2}+\sigma_{2}^{2}} \Phi^{-1}\left(t_{1}\right)\right), \quad q_{21}^{*}=\max \left\{0,(1+\gamma) \theta_{1}-q_{1}\right\}$ If $k_{2}+\sqrt{\sigma_{0}^{2}+\sigma_{2}^{2}} \Phi^{-1}\left(t_{1}\right)<\theta_{1}$, we take the lower bound of $\Omega_{1}\left(\theta_{1}\right)$ instead of $\left(k_{2}+\sqrt{\sigma_{0}^{2}+\sigma_{2}^{2}} \Phi^{-1}\left(t_{1}\right)\right)$, $q_{21}^{*}=\max \left\{0, \theta_{1}-q_{1}\right\}$. The proof of $\Omega_{2}$ is the same as $\Omega_{1}$.

\section{Appendix C}

Proof of Equations (20)-(25): From (18), when $k_{2}>q_{1}-\sqrt{\sigma_{0}^{2}+\sigma_{2}^{2}} \Phi^{-1}\left(t_{1}\right)$, we can know $q_{21}>0$; when $k_{2}>q_{1}-\sqrt{\sigma_{0}^{2}+\sigma_{2}^{2}} \Phi^{-1}\left(t_{1}\right), q_{21}=0$. For given $q_{1}, c^{(2)}$ and $k_{2}$, we can discuss $E_{(1)}\left[\Pi\left(q_{1}\right)\right]_{\left(q_{1}, q_{2}\right) \in \Omega_{1}}$ with two conditions: $q_{2}>0, q_{2}=0$ and expected profit at stage 2 can be expressed as follows:

$$
\begin{aligned}
& \text { when } q_{2}>0, k_{2}>q_{1}-\sqrt{\sigma_{o}^{2}+\sigma_{2}^{2}} \Phi^{-1}\left(t_{1}\right) \\
& E_{(2)}\left[\Pi\left(q_{2}>0, q_{1}\right)_{\left(q_{1}, q_{2}\right) \in \Omega_{1}}\right]=\int_{-\infty}^{\theta_{1}} p \theta_{1} f_{2}(x) \mathrm{d} x+\int_{\theta_{1}}^{k_{2}+\sqrt{\sigma_{0}^{2}+\sigma_{2}^{2}} \Phi^{-1}\left(t_{1}\right)} p x f_{2}(x) \mathrm{d} x \\
& +\int_{k_{2}+\sqrt{\sigma_{0}^{2}+\sigma_{2}^{2}} \Phi^{-1}\left(t_{1}\right)}^{\infty} P\left(k_{2}+\sqrt{\sigma_{0}^{2}+\sigma_{2}^{2}} \Phi^{-1}\left(t_{1}\right)\right) f_{2}(x) \mathrm{d} x-c_{h 1} \int_{-\infty}^{\theta_{1}}\left(\theta_{1}-x\right) f_{2}(x) \mathrm{d} x-c_{h 2} \int_{-\infty}^{\theta_{1}}\left(k_{2}+\sqrt{\sigma_{0}^{2}+\sigma_{2}^{2}} \Phi^{-1}\left(t_{1}\right)-\theta_{1}\right) f_{2}(x) \mathrm{d} x \\
& -c_{h 2} \int_{\theta_{1}}^{k_{2}+\sqrt{\sigma_{0}^{2}+\sigma_{2}^{2}} \Phi^{-1}\left(t_{1}\right)}\left(k_{2}+\sqrt{\sigma_{0}^{2}+\sigma_{1}^{2}} \Phi^{-1}\left(t_{1}\right)-x\right) f_{2}(x) \mathrm{d} x-c_{s 1} \int_{k_{2}+\sqrt{\sigma_{0}^{2}+\sigma_{2}^{2}} \Phi^{-1}\left(t_{1}\right)}^{(1+\gamma) \theta_{1}}\left(x-k_{2}-\sqrt{\sigma_{0}^{2}+\sigma_{2}^{2}} \Phi^{-1}\left(t_{1}\right)\right) f_{2}(x) \mathrm{d} x \\
& -c_{s 2} \int_{(1+\gamma) \theta_{1}}^{\infty}\left(x-(1+\gamma) \theta_{1}\right) f_{2}(x) \mathrm{d} x-c^{(2)}\left(k_{2}+\sqrt{\sigma_{0}^{2}+\sigma_{2}^{2}} \Phi^{-1}\left(t_{1}\right)-q_{1}\right)=\left(p-c_{h 1}+c_{h 2}\right) \sqrt{\sigma_{0}^{2}+\sigma_{2}^{2}} \Psi\left(\left(\theta_{1}-k_{2}\right) / \sqrt{\sigma_{0}^{2}+\sigma_{2}^{2}}\right) \\
& -\left(p+c_{h 2}+c_{s 1}\right) \sqrt{\sigma_{0}^{2}+\sigma_{2}^{2}} \Psi\left(\Phi^{-1}\left(t_{1}\right)\right)+\left(c_{s 1}-c_{s 2}\right) \sqrt{\sigma_{0}^{2}+\sigma_{2}^{2}} \Psi\left(\left((1+\gamma) \theta_{1}-k_{2}\right) / \sqrt{\sigma_{0}^{2}+\sigma_{2}^{2}}\right) \\
& +\left\{p-c_{h 1}+c_{h 2}+c_{s 1}\left[1-\Phi\left(\left((1+\gamma) \theta_{1}-k_{2}\right) / \sqrt{\sigma_{0}^{2}+\sigma_{2}^{2}}\right)\right]\right\} \theta_{1} \\
& +\left\{c_{h 1}-c_{h 2}-c^{(2)}-c_{s 1}\left[1-\Phi\left(\left((1+\gamma) \theta_{1}-k_{2}\right) / \sqrt{\sigma_{0}^{2}+\sigma_{2}^{2}}\right)\right]\right\} k_{2} \\
& -\left\{c^{(2)}+c_{h 2}+c_{s 1}\left[1-\Phi\left(\left((1+\gamma) \theta_{1}-k_{2}\right) / \sqrt{\sigma_{0}^{2}+\sigma_{2}^{2}}\right)\right]\right\} \sqrt{\sigma_{0}^{2}+\sigma_{2}^{2}} \Psi\left(\Phi^{-1}\left(t_{1}\right)\right) \\
& +c_{s 1} \gamma \theta_{1}\left[1-\Phi\left(\left((1+\gamma) \theta_{1}-k_{2}\right) / \sqrt{\sigma_{0}^{2}+\sigma_{2}^{2}}\right)\right]+c^{(2)} q_{1}
\end{aligned}
$$


when $q_{21}=0$,

$$
\begin{aligned}
& E_{(2)}\left[\Pi\left(q_{2}=0, q_{1}\right)_{\left(q_{1}, q_{2}\right) \in \Omega_{1}}\right]=\int_{-\infty}^{\theta_{11}} p \theta_{1} f_{2}(x) \mathrm{d} x+\int_{\theta_{1}}^{q_{1}} p x f_{2}(x) \mathrm{d} x+\int_{q_{1}}^{\infty} p q_{1} f_{2}(x) \mathrm{d} x-c_{h 2} \int_{-\infty}^{\theta_{1}}\left(\theta_{1}-x\right) f_{2}(x) \mathrm{d} x \\
& -c_{h 2} \int_{-\infty}^{\theta_{1}}\left(q_{1}-\theta_{1}\right) f_{2}(x) \mathrm{d} x-c_{h 2} \int_{\theta_{1}}^{q_{1}}\left(q_{1}-x\right) f_{2}(x) \mathrm{d} x-c_{s 1} \int_{q_{1}}^{(1+\gamma) \theta_{1}}\left(x-q_{1}\right) f_{2}(x) \mathrm{d} x \\
& -c_{s 2} \int_{(1+\gamma) \theta_{1}}^{\infty}\left(x-(1+\gamma) \theta_{1}\right) f_{2}(x) \mathrm{d} x=-\left(p+c_{h 2}+c_{s 1}\right) \sqrt{\sigma_{0}^{2}+\sigma_{2}^{2}} \Psi\left(\left(q_{1}-k_{2}\right) / \sqrt{\sigma_{0}^{2}+\sigma_{2}^{2}}\right) \\
& +\left(p-c_{h 1}+c_{h 2}\right) \sqrt{\sigma_{0}^{2}+\sigma_{2}^{2}} \Psi\left(\left(q_{1}-k_{2}\right) / \sqrt{\sigma_{0}^{2}+\sigma_{2}^{2}}\right)+\left(c_{s 1}-c_{s 2}\right) \sqrt{\sigma_{0}^{2}+\sigma_{2}^{2}} \Psi\left(\left((1+\gamma) \theta_{1}-k_{2}\right) / \sqrt{\sigma_{0}^{2}+\sigma_{2}^{2}}\right) \\
& +\left\{p-c_{h 1}+c_{h 2}+c_{s 1}\left[1-\Phi\left(\left((1+\gamma) \theta_{1}-k_{2}\right) / \sqrt{\sigma_{0}^{2}+\sigma_{2}^{2}}\right)\right]\right\} \theta_{1}-\left\{c_{h 2}+c_{s 1}\left[1-\Phi\left(\left((1+\gamma) \theta_{1}-k_{2}\right) / \sqrt{\sigma_{0}^{2}+\sigma_{2}^{2}}\right)\right]\right\} q_{1} \\
& +c_{s 1}\left[1-\Phi\left(\left((1+\gamma) \theta_{1}-k_{2}\right) / \sqrt{\sigma_{0}^{2}+\sigma_{2}^{2}}\right)\right] \gamma \theta_{1}+c_{h 1} k_{2}
\end{aligned}
$$

Therefore

$$
\begin{aligned}
E_{(1)}\left[\Pi\left(q_{1}\right)\right]_{\left(q_{1}, q_{2}\right) \in \Omega_{1}}= & \sum_{i=1}^{n} p_{i}\left(\int_{-\infty}^{q_{1}-\sqrt{d_{1}+d_{3}} \Phi^{-1}\left(t_{1}\right)} E_{(2)}\left[\Pi\left(q_{2}=0, q_{1}\right)_{\left(q_{1}, q_{2}\right) \in \Omega_{1}}\right] f_{K_{2}}\left(k_{2}\right) \mathrm{d} k_{2}\right. \\
& \left.+\int_{q_{1}-\sqrt{d_{1}+d_{3}} \Phi^{-1}\left(t_{1}\right)}^{\infty} E_{(2)}\left[\Pi\left(q_{2}>0, q_{1}\right)_{\left(q_{1}, q_{2}\right) \in \Omega_{1}}\right] f_{K_{2}}\left(k_{2}\right) \mathrm{d} k_{2}-c^{(1)} q_{1}\right)
\end{aligned}
$$

By the same discussions, $E_{(1)}\left[\Pi\left(q_{1}\right)\right]_{\left(q_{1}, q_{2}\right) \in \Omega_{2}}$ can be expressed as follows:

when $q_{2}>0, k_{2}>q_{1}-\sqrt{\sigma_{0}^{2}+\sigma_{2}^{2}} \Phi^{-1}\left(t_{2}\right)$

$$
\begin{aligned}
& E_{(2)}\left[\Pi\left(q_{2}>0, q_{1}\right)_{\left(q_{1}, q_{2}\right) \in \Omega_{1}}\right]=\int_{-\infty}^{\theta_{1}} p \theta_{1} f_{X_{2}}\left(x_{2}\right) \mathrm{d} x_{2}+\int_{\theta_{1}}^{k_{2}+\sqrt{\sigma_{0}^{2}+\sigma_{2}^{2}} \Phi^{-1}\left(t_{2}\right)} p x f_{2}(x) \mathrm{d} x \\
& +\int_{k_{2}+\sqrt{\sigma_{0}^{2}+\sigma_{2}^{2}} \Phi^{-1}\left(t_{2}\right)}^{\infty} p\left(k_{2}+\sqrt{\sigma_{0}^{2}+\sigma_{2}^{2}} \Phi^{-1}\left(t_{2}\right)\right) f_{2}(x) \mathrm{d} x-c_{h 1} \int_{-\infty}^{\theta_{1}}\left(\theta_{1}-x\right) f_{2}(x) \mathrm{d} x \\
& -c_{h 2} \int_{-\infty}^{\theta_{1}}\left(k_{2}+\sqrt{\sigma_{0}^{2}+\sigma_{2}^{2}} \Phi^{-1}\left(t_{2}\right)-\theta_{1}\right) f_{2}(x) \mathrm{d} x-c_{h 2} \int_{\theta_{1}}^{k_{2}+\sqrt{\sigma_{0}^{2}+\sigma_{2}^{2}} \Phi^{-1}\left(t_{2}\right)}\left(k_{2}+\sqrt{\sigma_{0}^{2}+\sigma_{2}^{2}} \Phi^{-1}\left(t_{2}\right)-x\right) f_{2}(x) \mathrm{d} x \\
& -c_{s 2} \int_{k_{2}+\sqrt{\sigma_{0}^{2}+\sigma_{2}^{2}} \Phi^{-1}\left(t_{2}\right)}^{\infty}\left(x_{2}-k_{2}-\sqrt{\sigma_{0}^{2}+\sigma_{2}^{2}} \Phi^{-1}\left(t_{2}\right)\right) f_{2}(x) \mathrm{d} x-c^{(2)}\left(k_{2}+\sqrt{\sigma_{0}^{2}+\sigma_{2}^{2}} \Phi^{-1}\left(t_{2}\right)-q_{1}\right) \\
& =\left(p-c_{h 1}+c_{h 2}\right) \sqrt{\sigma_{0}^{2}+\sigma_{2}^{2}} \Psi\left(\left(\theta_{1}-k_{2}\right) / \sqrt{\sigma_{0}^{2}+\sigma_{2}^{2}}\right)-\left(p+c_{h 2}+c_{s 2}\right) \sqrt{\sigma_{0}^{2}+\sigma_{2}^{2}} \Psi\left(\Phi^{-1}\left(t_{2}\right)\right) \\
& +\left(p-c_{h 1}+c_{h 2}\right) \theta_{1}+\left(c_{h 1}-c_{h 2}-c_{2}\right) k_{2}-\left(c^{(2)}+c_{h 2}\right) \sqrt{\sigma_{0}^{2}+\sigma_{2}^{2}} \Psi\left(\Phi^{-1}\left(t_{2}\right)\right)+c^{(2)} q_{1}
\end{aligned}
$$

when $q_{22}=0$,

$$
\begin{aligned}
& E_{(2)}\left[\Pi\left(q_{2}=0, q_{1}\right)_{\left(q_{1}, q_{2}\right) \in \Omega_{2}}\right]=\int_{-\infty}^{\theta_{1}} p \theta_{1} f_{2}(x) \mathrm{d} x+\int_{\theta_{1}}^{q_{1}} p x f_{2}(x) \mathrm{d} x+\int_{q_{1}}^{\infty} p q_{1} f_{2}(x) \mathrm{d} x \\
& -c_{h 1} \int_{-\infty}^{\theta_{1}}\left(\theta_{1}-x\right) f_{2}(x) \mathrm{d} x-c_{h 2} \int_{-\infty}^{\theta_{1}}\left(q_{1}-\theta_{1}\right) f_{2}(x) \mathrm{d} x-c_{h 2} \int_{\theta_{1}}^{q_{1}}\left(q_{1}-x\right) f_{2}(x) \mathrm{d} x-c_{s 2} \int_{q_{1}}^{\infty}\left(x-q_{1}\right) f_{2}(x) \mathrm{d} x \\
& =-\left(p+c_{h 2}+c_{s 2}\right) \sqrt{\sigma_{0}^{2}+\sigma_{2}^{2}} \Psi\left(\left(q_{1}-k_{2}\right) / \sqrt{\sigma_{0}^{2}+\sigma_{2}^{2}}\right)+\left(p-c_{h 1}+c_{h 2}\right) \sqrt{\sigma_{0}^{2}+\sigma_{2}^{2}} \Psi\left(\left(\theta_{1}-k_{2}\right) / \sqrt{\sigma_{0}^{2}+\sigma_{2}^{2}}\right) \\
& +\left(p-c_{h 1}+c_{h 2}\right) \theta_{1}+c_{h 1} k_{2}-c_{h 2} q_{1}
\end{aligned}
$$

Therefore 


$$
\begin{aligned}
E_{(1)}\left[\Pi_{2}\left(q_{1}\right)\right]_{\left(q_{1}, q_{2}\right) \in \Omega_{2}}= & \sum_{i=1}^{n} p_{i}\left(\int_{-\infty}^{q_{1}-\sqrt{d_{1}+d_{3}} \Phi^{-1}\left(t_{2}\right)} E_{(2)}\left[\Pi\left(q_{2}=0, q_{1}\right)_{\left(q_{1}, q_{2}\right) \in \Omega_{2}}\right] f_{K_{2}}\left(k_{2}\right) \mathrm{d} k_{2}\right. \\
& \left.+\int_{q_{1}-\sqrt{d_{1}+d_{3}} \Phi^{-1}\left(t_{2}\right)}^{\infty} E_{(2)}\left[\Pi\left(q_{2}>0, q_{1}\right)_{\left(q_{1}, q_{2}\right) \in \Omega_{2}}\right] f_{K_{2}}\left(k_{2}\right) \mathrm{d} k_{2}-c^{(1)} q_{1}\right)
\end{aligned}
$$

\section{Appendix D}

\section{Proof of Proposition 4:}

In $\Omega_{1}$, we redefine $\partial E_{(1)}\left[\Pi\left(q_{1}\right)\right]_{\left(q_{1}, q_{2}\right) \in \Omega_{1}} / \partial q_{1}=G\left(q_{1}\right)$. If $\bar{c}^{(2)}-c^{(1)} \geq 0, G\left(q_{1}\right)=\bar{c}^{(2)}-c^{(1)} \geq 0$ as $q_{1} \rightarrow-\infty$ and $G\left(q_{1}\right)=-c_{h 1}-c^{(1)}<0$ as $q_{1} \rightarrow \infty$, by the intermediate value theorem, there exists a $q_{11} \in[-\infty, \infty]$ such that $\partial E_{(1)}\left[\Pi\left(q_{1}\right)\right]_{\left(q_{1}, q_{2}\right) \in \Omega_{1}} / \partial q_{1}=0$. If $\bar{c}^{(2)}-c^{(1)}<0$, $G\left(q_{1}\right)<0$ as $q_{1} \rightarrow-\infty$ and $G\left(q_{1}\right)<0$ as $q_{1} \rightarrow \infty$, hence $q_{1}=0$ when $\bar{c}^{(2)}-c^{(1)}<0$.
In $\Omega_{2}$, we redefine $\partial E_{(1)}\left[\Pi\left(q_{1}\right)\right]_{\left(q_{1}, q_{2}\right) \in \Omega_{2}} / \partial q_{1}=G\left(q_{1}\right)$, if $\bar{c}^{(2)}-c^{(1)} \geq 0, \quad G\left(q_{1}\right)=\bar{c}^{(2)}-c^{(1)} \geq 0 \quad$ as $\quad q_{1} \rightarrow-\infty$ and $G\left(q_{1}\right)=-c_{h 2}-c^{(1)}<0$ as $q_{1} \rightarrow \infty$, by the intermediate value theorem, there exists a $q_{12} \in[-\infty, \infty]$ such that $\partial E_{(1)}\left[\Pi\left(q_{1}\right)\right]_{\left(q_{1}, q_{2}\right) \in \Omega_{2}} / \partial q_{1}=0$. If $\bar{c}^{(2)}-c^{(1)}<0$, $G\left(q_{1}\right)<0$ as $q_{1} \rightarrow-\infty$ and $G\left(q_{1}\right)<0$ as $q_{1} \rightarrow \infty$, hence $q_{1}=0$ when $\bar{c}^{(2)}-c^{(1)}<0$ and this completes the proof. 